\title{
Effects of climate change on an emperor penguin population: analysis of coupled demographic and climate models.
}

\author{
Stéphanie Jenouvrier ${ }^{1,2,4,5}$, \\ Marika Holland ${ }^{3}$, \\ Julienne Strove ${ }^{4}$, \\ Christophe Barbraud ${ }^{2}$, \\ Henri Weimerskirch ${ }^{2}$, \\ Mark Serreze ${ }^{4,5}$, \\ Hal Caswell ${ }^{1,6}$
}

June 21, 2012

Running title: Emperor penguins and climate change

To whom correspondence should be addressed to sjenouvrier@whoi.edu. phone:+1 5082893245

Emails of co-authors: mholland@ucar.edu; stroeve@kryos.colorado.edu; barbraud@cebc.cnrs.fr; henriw@cebc.cnrs.fr; serreze@kryos.colorado.edu; hcaswell@whoi.edu.

1. Biology Dept., MS-34, Woods Hole Oceanographic Institution, Woods Hole, MA 02543, USA

2. Centre d'Etudes Biologiques de Chizé, Centre National de la Recherche Scientifique, F-79360 Villiers en Bois, France

3. Oceanography Section, National Center for Atmospheric Research, Boulder, 80305 CO, USA

4. National Snow and Ice Data Center, Boulder, 80309 CO, USA

5. Cooperative Institute for Research in Environmental Science, University of Colorado Boulder, 80309-0449, USA

6. Max Planck Institute for Demographic Research, Rostock, Germany.

Keywords: stochastic matrix population model; stochastic climate forecast; IPCC; uncertainties; sea ice; seabirds. 
1 The following table of contents is provided for convenience of reviewers.

\section{Contents}

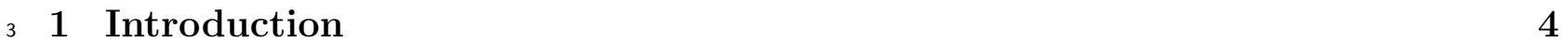

$\begin{array}{llll}4 & 2 & \text { Emperor penguin life cycle and sea ice } & 6\end{array}$

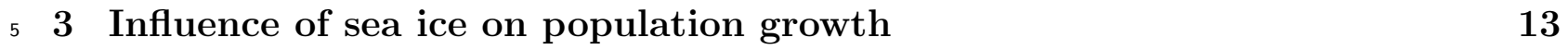

64 Population response to climate change $\quad 15$

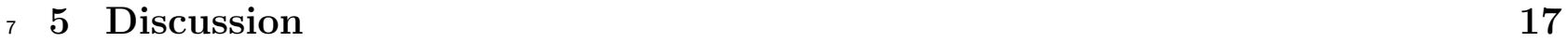

$\begin{array}{llll}8 & 6 & \text { Acknowledgments } & 26\end{array}$

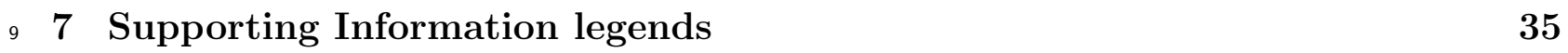

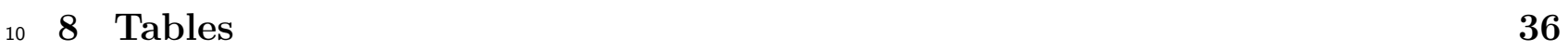

$\begin{array}{llll}11 & 9 & \text { Figures } & 41\end{array}$ 


\begin{abstract}
Sea ice conditions in the Antarctic affect the life cycle of the emperor penguin (Aptenodytes forsteri). We present a population projection for the emperor penguin population of Terre Adélie, Antarctica, by linking demographic models (stagestructured, seasonal, nonlinear, two-sex matrix population models) to sea ice forecasts from an ensemble of IPCC climate models. Based on maximum likelihood capturemark-recapture analysis, we find that seasonal sea ice concentration anomalies $\left(S I C_{a}\right)$ affect adult survival and breeding success. Demographic models show that both deterministic and stochastic population growth rates are maximized at intermediate values of annual $S I C_{a}$, because neither the complete absence of sea ice, nor heavy and persistent sea ice, would provide satisfactory conditions for the emperor penguin. We show that under some conditions the stochastic growth rate is positively affected by the variance in $S I C_{a}$. We identify an ensemble of 5 general circulation climate models whose output closely matches the historical record of sea ice concentration in Terre Adélie. The output of this ensemble is used to produce stochastic forecasts of $S I C_{a}$, which in turn drive the population model. Uncertainty is included by incorporating multiple climate models and by a parametric bootstrap procedure that includes parameter uncertainty due to both model selection and estimation error. The median of these simulations predicts a decline of the Terre Adélie emperor penguin population of $81 \%$ by the year 2100 . We find a $43 \%$ chance of an even greater decline, of $90 \%$ or more. The uncertainty in population projections reflects large differences among climate models in their forecasts of future sea ice conditions. One such model predicts population increases over much of the century, but overall, the ensemble of models predicts that population declines are far more likely than population increases. We conclude that climate change is a significant risk for the emperor penguin. Our analytical approach, in which demographic models are linked to IPCC climate models, is powerful and generally applicable to other species and systems.
\end{abstract}




\section{Introduction}

Given the observed and predicted changes in climate (Solomon et al., 2007), conservation biologists face a major challenge because climate affects all aspects of the life cycle of a species (life history traits, phenology, movement). This results in changes in populations, species distributions, and ecosystems (see reviews by Hughes (2000); McCarty (2001); Parmesan (2006); Parmesan \& Yohe (2003); Vitousek (1994); Walther et al. (2002)). The emperor penguin (Aptenodytes forsteri) is a species that is known to be extremely sensitive to climate change, especially to changes in the sea ice environment (Ainley et al., 2010; Barbraud \& Weimerskirch, 2001; Croxall et al., 2002; Forcada \& Trathan, 2009; Jenouvrier et al., 2005a, 2009b; Trathan et al., 2011; Fretwell \& Trathan, 2009). In this paper, we analyze the population responses of the emperor penguin to sea ice conditions and project its fate over the rest of the 21st century using a novel and more comprehensive analysis than previous studies.

Climate may affect vital rates (a general term for rates of survival, fertility, development, etc.) in many ways (Ballerini et al., 2009; Stenseth et al., 2002) and possibly in opposite directions (Barbraud \& Weimerskirch, 2001). For example, mean winter temperature has a positive effect on adult survival but a negative effect on fecundity of the Eurasian Oystercatcher (Haematopus ostralegus) (van de Pol et al., 2010). Therefore, to fully understand the population effects of climate change, climate effects on the vital rates should ideally be incorporated into models including the full life cycle (Adahl et al., 2006; van de Pol et al., 2010). Although many studies have related climate to one or a few vital rates (e.g. survival Jenouvrier et al. (2005b, 2003)), few have integrated these effects into the entire life cycle (but see e.g. Jenouvrier et al. (2009b); Wolf et al. (2010); Hunter et al. (2010); Barbraud et al. (2010)).

Seasonality can be particularly important, because the effects may occur at different times during the seasonal cycle (Visser et al., 1998). Complex interactions of climate variables have been shown to occur for seasonally breeding species, which may fail to adjust their breeding 
phenology to track the peak of food availability for their young (Both et al., 2006; Moller et al., 2008).

The responses of vital rates to climate conditions is only half of the story; to project the population response to climate change, it is necessary to link the demographic models to forecasts of future conditions (e.g. Jenouvrier et al. (2009b)). A primary source for such forecasts is the set of climate model simulations that have contributed to the Intergovernmental Panel on Climate Change (IPCC) assessment reports (Stock et al., 2011). A growing number of studies have now linked climate-dependent demographic models to these climate models (e.g. seabirds Jenouvrier et al. (2009b); Barbraud et al. (2010); polar bears Hunter et al. (2010)).

Our approach is to measure the effect of climate on the vital rates in a complete life cycle model, to incorporate those vital rates into a population model to compute population growth as a function of climate, and then to obtain forecasts of climate trajectories from climate models and use those to drive projections of population growth. In this paper, we extend previous studies (Barbraud and Weimerskirch 2001, Jenouvrier et al. 2005b, 2009b, 2010), especially that of Jenouvrier et al. (2009b), in several ways. (1) We obtain rigorous statistical estimates of how sea ice, at different seasons of the year, affects penguin vital rates. (2) We distinguish males and females, recognizing that the sexes differ in their sensitivity to sea ice variations (Jenouvrier et al. 2005b) and that breeding is absolutely dependent on participation by both males and females (Prevost, 1961). (3) We introduce a new method of selecting climate models based on the agreement of their output with both the mean and the variance in observed sea ice. (4) In order to include stochasticity in the climate forecasts, only one or a few realizations of which are available, we developed a new method to estimate stochasticity from time series of global circulation model (GCM) output.

Projections from models that are estimated from data are always accompanied by uncertainty. If the results are to be useful for policy makers, it is essential to quantify that uncertainty, and to draw conclusions that remain valid even given the range of uncertainty 
(Hunter et al. 2010). We have used the results of statistical estimation of demographic parameters, and differences among an ensemble of climate models, to quantify this uncertainty.

The organization of the paper is as follows. In Section 2 we use a long-term dataset to estimate the effects of sea ice on the vital rates. In Section 3 we evaluate the effect of sea ice on deterministic and stochastic population growth rates for the emperor penguin. In Section 4, we compute stochastic sea ice forecasts from a selected set of climate models, and use those forecasts to project population response to future sea ice change. We conclude with discussion in Section 5.

\section{Emperor penguin life cycle and sea ice}

Various components of the sea ice environment affect different parts of the emperor penguin life cycle during different seasons (see review by Croxall et al. (2002); Forcada \& Trathan (2009); Ainley et al. (2010)). In this section we outline the life cycle of the emperor penguin and define the sea ice variables used in our analysis. Then we discuss the mechanisms by which sea ice affects the vital rates and present the results of estimating these effects using capture-mark-recapture (CMR) analysis.

\section{Study population and data}

Our analysis is based on a long-term data set on breeding emperor penguins at Dumont D'Urville, Terre Adélie, in Antarctica ( $\left.66^{\circ} 40 \mathrm{~S} 140^{\circ} 01 \mathrm{E}\right)$. The colony has been monitored every year, during the breeding season (March-December), from 1962 onwards. For details of the history and data, see Prevost (1961) and Jenouvrier et al. (2005a). From 1962 onwards, breeding adults, number of eggs, frozen chicks, and surviving chicks at the end of the breeding season have been counted, allowing the estimation of breeding success (Barbraud \& Weimerskirch, 2001). From 1968 to 1988, penguins were individually marked using flipper bands. Banding stopped in 1988, but banded birds have been recorded since then. We limit our analysis to the period before 2000 because too few marked birds were seen after that to permit estimation. 


\section{The life cycle and the demographic model}

Emperor penguins breed on motionless sea ice (i.e. fast ice) during the Antarctic winter. They arrive at the colony sometime in late March to early April while sea ice is thickening, and leave the colony in late December before the ice breaks up. The colony site is usually far from the ocean, and during the breeding season emperor penguins travel long distances to feed in ice-free areas, such as polynyas, within the sea ice cover. They feed on fish (mainly Pleuragramma antarcticum), crustaceans (mainly Euphausia superba and amphipods), and squid (mainly Psychroteuthis glacialis) (Cherel, 2008; Cherel \& Kooyman, 1998; Kirkwood \& Robertson, 1997). The female lays a single egg in May, which is then incubated by the male for two months while the female leaves the colony to feed. Females return when chicks hatch around July, and both parents take turns feeding the chick until fledging in December.

Our demographic model is described in detail in Jenouvrier et al. (2010). It is a stageclassified matrix population model with a projection interval of one year, but the annual projection is based on four seasonal steps. The model has 5 stages: male and female prebreeders (birds that have yet to breed for the first time), breeding pairs, and male and female non-breeders (birds that have bred before but do not do so in the current year). The formation of pairs is a nonlinear function of the operational sex ratio.

Our model does not include density dependence because we expect small density effects in this population relative to effects of environmental variations, and especially sea ice (Supplementary Appendix S1, Herrando-Pérez et al. (2012)).

The annual population projection depends on the vital rates: the probability that an individual of a given stage returns to the breeding site, the probability of mating as a function of the availability of potential mates, breeding success (probability that a breeding pair raises offspring given the female lays an egg), the primary sex ratio (fixed at 0.5 ), the survival of offspring during the first year at sea, and the annual survival of pre-breeders, non-breeders and male and female breeders.

We divide the year into four seasons: (1) the non-breeding season from January to March, 
(2) the arrival, copulation and laying period (April-May), hereafter called the laying period,

(3) the incubation period (June-July) and, (4) the rearing period (August-December).

\section{Sea ice variables}

Many components of the sea ice environment affect penguins in various way. To avoid examining the effect of all possible factors on vital rates and to untangle the networks of causation among them, we examine the covariation among several factors selected on the basis on the emperor penguin responses to climate (i.e fast ice area and polynya area indices, sea ice concentration (SIC) and sea ice extent (SIE), see Ainley et al. 2010 for a comprehensive review). All these variables are strongly correlated (Supplementary Appendix S2) and we focus our analysis on SIC, including the seasonality in SIC because it drives the emperor penguin life cycle.

Sea ice concentration is the fraction of area covered by sea ice. Observed values of SIC from 1979 to 2007 were obtained from passive microwave satellite imagery provided by the National Snow and Ice data Center, using the NASA Team sea ice algorithm (see Cavalieri et al. (1996) and http://nsidc.org/data). Forecasts of SIC from climate models were extracted from 20 models available as part as the WCRP CMIP3 multi-model dataset from 1900 to 2100 (see Meehl et al. (2007) and http://esg.1lnl.gov/portal).

In order to link population models to the output of GCMs, which use relatively coarse spatial grids (100-200 km resolution), we use observed values of SIC over similarly large spatial scales. We averaged SIC, both observed and simulated, over a large sector centered on the colony. This sector included a 20 degree span in latitude, between longitudes $130^{\circ} \mathrm{E}$ and $150^{\circ} \mathrm{E}$ during the breeding season, and between longitudes $120^{\circ} \mathrm{E}$ and $160^{\circ} \mathrm{E}$ during the nonbreeding season. This includes the maximum foraging distances from the colony, of about $100 \mathrm{~km}$ during the breeding season and at least $650 \mathrm{~km}$ during the non-breeding season (Zimmer et al. 2008).

As a variable to describe the sea ice conditions, we use the proportional anomalies in SIC, relative to the mean from 1979 to 2007 . We denote this variable as $S I C_{a}$, and calculate it for 
each of the four seasons (Fig. 1). We estimated the vital rates as functions of the observed seasonal $S I C_{a}$ (see following section), and used forecasts of seasonal $S I C_{a}$ to project future population trajectories (Section 4). However, to provide a comprehensive understanding of the effect of seasonal $S I C_{a}$ on vital rates and population growth rate, we present our results as functions of "annual sea ice" and "seasonal difference in sea ice", two variables that accounted for most of the variability in the four seasonal $S I C_{a}$ variables (Fig. 2, S2). Annual sea ice is a weighted mean of seasonal $S I C_{a}$ over the year. The seasonal difference in $S I C_{a}$ is the difference between ice concentration in the non-breeding season and its weighted average over the combined incubation and rearing seasons. The contribution of $S I C_{a}$ variations during the laying season to the seasonal $S I C_{a}$ difference variations is small. Thus positive values of the seasonal $S I C_{a}$ difference correspond to years with positive SIC anomalies in the non-breeding season and negative SIC anomalies in the incubating and rearing seasons.

\section{Relationships between sea ice and vital rates}

Sea ice concentration affects emperor penguin vital rates through various mechanisms, which are not mutually exclusive; see review by Ainley et al. (2010). First, SIC may directly affect foraging; in years with dense sea ice cover, foraging trips to the nearest open water area are on average longer, energetic costs for breeding adults are higher, and the provisioning of chicks is lower (Massom et al., 2009; Zimmer et al., 2008). We would thus expect negative effects of high $S I C_{a}$ on breeding success and on adult survival of both sexes during the rearing period.

Sea ice concentration is also critical to Antarctic ecosystem function (Thomas \& Dieckmann, 2003). It may indirectly affect the emperor penguin through its effects on other species of the Antarctic food web, either prey or predators (Ainley et al., 2010, 2007; Barbraud \& Cotte, 2008; Barbraud \& Weimerskirch, 2001; Forcada \& Trathan, 2009). However, the mechanisms involved remain unclear and their relative importance is still debated (Ainley et al., 2007; Barbraud \& Cotte, 2008).

The effects of SIC on primary productivity and krill may cascade up to fish and upper 
predators; e.g. Nicol et al. (2000); Forcada \& Trathan (2009); Fraser \& Hoffmann (2003). Primary productivity and krill density are known to be related to sea ice extent (SIE) and concentration (Atkinson et al., 2004; Brierley et al., 2002; Loeb et al., 1997). Around Antarctica, krill density during summer is positively related to chlorophyll concentrations (Atkinson et al., 2008). In the South West Atlantic, krill densities and recruitment during summer are positively related to SIE of the previous winter (Loeb et al., 1997; Nicol et al., 2000; Atkinson et al., 2004). Other studies have shown non-linear relations between sea ice cover and krill populations (Quetin et al., 2007; Wiedenmann et al., 2009). High krill recruitment occurs over a range of optimum sea ice conditions, suggesting complex mechanisms linking sea ice and krill abundance.

Sea ice may also influence the emperor penguin by top-down processes (Ainley et al., 2010, 2007). Reduced sea ice cover may allow greater access to the foraging areas of the emperor penguin by potential predators such as killer whales (Orcinus orca (Ainley et al., 2010, 2007; Pitman \& Durban, 2010), although no emperor penguin remains have been detected in the diet of killer whales (Barbraud \& Cotte, 2008). Energetically compromised penguins (especially males after their four months fast) may be particularly vulnerable to predation (Ainley personal communication).

\section{Parameter estimation}

The capture-recapture dataset permits the estimation of survival and return probabilities (see Jenouvrier et al. (2005a, 2010) for more details), which may differ between males and females because of their different breeding investment (Barbraud \& Weimerskirch, 2001; Jenouvrier et al., 2005a). We estimated probabilities of survival, and of return to the breeding colony, using a multistate capture-mark-recapture model (CMR) based on sex and reproductive status, with some unobservable stages. Adult survival is allowed to differ between sexes, and return probability to differ between breeders and non-breeders. The statistical capture-recapture model is described in detail in Appendix C of Jenouvrier et al. (2010); for a review of these methods see Lebreton et al. (2009). 
We estimated linear and quadratic effects of $S I C_{a}$ using models relating the vital rates to covariates with a general logit link function (Lebreton et al., 1992). It is impossible to test the impact of $S I C_{a}$ on survival during the first year at sea, or on the survival or return probabilities of pre-breeders, because data on pre-breeders and $S I C_{a}$ overlap for only a few years (modern SIC satellite data are only available from 1979 onwards).

Estimation and CMR model selection was performed using the program M-SURGE (Choquet et al., 2004). We used Akaike's information criterion (AIC, Akaike (1974)) to compare models, the model with the lowest AIC being the model most supported by the data. We based our inferences on the most plausible set of models using model averaging using AIC weights $w_{i}$ (Burnham \& Anderson, 2002). Thus a parameter $\theta$ is calculated as

$$
\theta=\operatorname{logit}^{-1}\left(\sum_{i=0}^{M} w_{i} \beta_{i}\right)
$$

where $\mathrm{M}$ is the number of models and $\beta_{i}$ is the estimated parameter on the logit scale for model $i$. The Akaike weights were calculated as

$$
w_{i}=\frac{\exp \left(-\Delta_{i} / 2\right)}{\sum_{r=0}^{M} \exp \left(-\Delta_{r} / 2\right)}
$$

where $\Delta_{i}=A I C_{i}-\min A I C$, where min $A I C$ is the smallest value of AIC in the model set.

Concerns have been raised that the use of flipper bands negatively affects the survival and breeding success of other species of penguins (Saraux et al., 2011). The banding study at Terre Adélie was discontinued in 1988 partly out of such concerns. However, the breeding success of banded and unbanded emperor penguins at Terre Adélie is not significantly different (Barbraud, unpublished results). Saraux et al. (2011) found that the effects of bands on survival of king penguins disappeared 4.5 years after banding. To reduce possible impacts of the flipper bands, but still retain sufficient sample size, we eliminated capture histories for the first two years after banding. Circumstantial evidence that the possible effects of 
bands are minimal in this population comes from estimates of population growth rate. If the banded birds on which CMR estimates are based had lower survival probabilities, we would expect estimated population growth rates to be consistently lower than those observed. This is not the case: the population growth rate obtained from CMR estimates agrees well with the observed population growth rate (Jenouvrier et al., 2010).

To quantify uncertainties resulting from model selection and estimation error, we used the parametric bootstrap procedure introduced by Regehr et al. (2010) and Hunter et al. (2010). To generate a bootstrap sample of a model output in this procedure, a CMR model is first selected with probability proportional to its AIC weight. Then a vector of parameter values is drawn from a multivariate normal distribution with a mean equal to the estimated parameter vector and a covariance matrix obtained from the Hessian matrix of the CMR model, or from the logistic regression model in the case of breeding success. The resulting parameter vector is used to create the population model, and the output calculated from that model. This process is repeated to generate a bootstrap sample, from which confidence intervals can be calculated using the percentile method.

\section{Results}

Supplementary Appendix 3 details some of the results outlined here, including the underlying parameter estimates and their uncertainties.

Breeding success is a decreasing function of $S I C_{a}$ during the rearing period (Figure 3). The CMR model selection procedure reveals effects of $S I C_{a}$ on adult survival of both sexes during all four seasons (Table 1). The models with $\triangle A I C \leq 4$ include effects in all seasons, those with $\triangle A I C \leq 3$ include effects in the laying, incubation, and rearing seasons, and those with $\triangle A I C \leq 2$ include effects during the incubation and rearing seasons. There was no support for effects of $S I C_{a}$ on the probability of return to the breeding colony (S3).

Figure 4 shows adult survival as a function of annual $S I C_{a}$ and seasonal differences in $S I C_{a}$. Survival probability is a concave nonlinear function of annual $S I C_{a}$ (Figure 4.a,b). The maximum annual adult survival is higher for females than for males (0.96 and 0.93 
respectively). The effect of seasonal differences in $S I C_{a}$ (Figure 4.c,d) is small compared to the effect of annual SICa, but it has an important effect on the difference between male and female survival. The effect of seasonal differences are positive when annual $S I C a<0$, and negative when annual $S I C_{a}>0$.

\section{Influence of sea ice on population growth}

To assess the effect of sea ice conditions on population growth, we use a demographic model in which the parameters are functions of $S I C_{a}$. Because sea ice is strongly seasonal and breeding biology is tied to the seasons, we use a seasonal periodic matrix model (Caswell 2001, Chap. 13) to capture these effects. The model, which is described in detail in Jenouvrier et al. (2010), includes a sequence of seasonal behaviors (arrival to the colony, mating, breeding) and accounts for differences in adult survival between males and females. The model is nonlinear because mating probability depends on the availability of males and females for mating. This frequency dependence is captured by expressing reproduction as a function of the proportional structure of the population.

In the model, a matrix $\mathbf{M}_{i}$ projects the population from season $i$ to season $i+1$. Since we identify four seasons, $\mathbf{M}_{4}$ projects the population from season 4 to season 1 in the next year. The annual projection matrix is given by the periodic product of the $\mathbf{M}_{i}$ :

$$
\mathbf{A}=\mathbf{M}_{4} \mathbf{M}_{3} \mathbf{M}_{2} \mathbf{M}_{1}
$$

where $\mathbf{M}_{1}$ includes the birth process, $\mathbf{M}_{2}$ includes annual mortality process, $\mathbf{M}_{3}$ includes migration to the breeding site, and $\mathbf{M}_{4}$ includes the mating process (Jenouvrier et al. 2010).

To establish clear notation, let $\mathbf{x}(t)$ represent the $S I C_{a}$ values (in all four seasons) in year $t$, and let $\mathbf{p}(t)$ be the proportional population structure in year $t$. The vector of demographic parameters is a function of sea ice and population composition; we denote it by $\boldsymbol{\theta}(\mathbf{x}, \mathbf{p})$. Then 
the population in year $t$ grows according to

$$
\mathbf{n}(t+1)=\mathbf{A}[\boldsymbol{\theta}(\mathbf{x}(t), \mathbf{p}(t))] \mathbf{n}(t)
$$

\section{Deterministic population growth}

In a fixed, specified sea ice environment, the population will eventually converge to an equilibrium proportional structure $\hat{\mathbf{p}}$ and (even though it is nonlinear) grow exponentially at a rate $\hat{\lambda}$ given by the dominant eigenvalue of the projection matrix evaluated at the equilibrium structure, $\mathbf{A}[\boldsymbol{\theta}(\mathbf{x}, \hat{\mathbf{p}})]$. To compute $\hat{\mathbf{p}}$, we calculate $S I C_{a}$, during each of the four seasons, from the annual $S I C_{a}$ and the seasonal differences in $S I C_{a}$. We then project the population from an arbitrary initial vector until it converges to the equilibrium structure, and use that structure to compute $\hat{\lambda}$. For a fixed value of annual $S I C_{a}$, the population growth rate increases (annual $S I C_{a}<-0.8$ ) or decreases (annual $S I C_{a}>-0.3$ ) with increasingly positive seasonal differences. Figure 5 shows $\hat{\lambda}$ as a function of annual $S I C_{a}$ and of seasonal differences in $S I C_{a}$. The population growth rate is maximized at intermediate values of $S I C_{a}$ close to 0 , and declines at higher or lower values.

The range of positive growth is wide (white contours on Figure 5) and $\hat{\lambda}$ declines from its maximum more rapidly for negative than for positive annual $S I C_{a}$ values.

\section{Stochastic population growth}

To examine the effect of sea ice variability, we calculate the stochastic population growth rate as a function of the means and variances of annual $S I C_{a}$ and of seasonal differences in $S I C_{a}$. The growth rates are calculated from stochastic simulations. At each time step $t$, values for annual $S I C_{a}$ and seasonal differences in $S I C_{a}$ are drawn from normal distributions with specified means and variances, and are used to parameterize the projection matrix $\mathbf{A}$ in eq.(3). We also include stochastic variation, unrelated to sea ice conditions, in breeding success and the probability of return to the colony. For breeding success, we add a normally distributed error term with variance given by the residual variation around the logistic re- 
gression of breeding success on $S I C_{a}$ (Figure 3 ). For probability of return, which is not a function of $S I C_{a}$, we sample repeatedly from the set of measured return probabilities from 1970 to 2000.

The simulation begins with an arbitrary population vector $\mathbf{n}_{0}$, and projects the population according to

$$
\mathbf{n}(t+1)=\mathbf{A}[\boldsymbol{\theta}(\mathbf{x}(t), \mathbf{p}(t))] \mathbf{n}(t)
$$

The stochastic growth rate is given by

$$
\log \lambda_{s}=\lim _{T \rightarrow \infty} \frac{1}{T} \log \|\mathbf{A}[\boldsymbol{\theta}(T-1)] \cdots \mathbf{A}[\boldsymbol{\theta}(0)] \mathbf{p}(0)\|
$$

We evaluate $\log \lambda_{s}$ numerically using $T=50,000$.

Figure 6 shows the results and Appendix S4 provides more details on the distribution of sea ice, vital rates and population growth rate for various examples. The stochastic growth rate is maximized at intermediate values of mean annual $S I C_{a}$, and declines as anomalies become very positive or very negative. At very high or very low values of the mean annual $S I C_{a}$ the growth rate is improved by increasing variance, but at intermediate values, the effect of variance is negative. The effect of the mean and variance in seasonal differences in $S I C_{a}$ are smaller, and negative. Changes in the mean and/or the variance of annual $S I C_{a}$ have the potential to greatly reduce the stochastic growth rate.

\section{Population response to climate change}

To project the population response to climate change, we use our demographic model to determine the response of the population to future sea ice conditions as forecasted by a select set of GCMs. We obtain forecasts of $S I C_{a}$ from a set of GCMs, compute stochastic $S I C_{a}$ forecasts from these, and use the results to generate population trajectories from 2000 to 2100 . 


\section{Stochastic sea ice forecasts}

GCMs differ in their ability to reproduce sea ice conditions in Antarctica. Thus, from an initial set of 20 climate models (Table 2), we selected those for which the statistical properties of the distribution of $S I C_{a}$ output agree well with the observations from 1979 to 2007, in terms of both the median and the standard deviation of the $S I C_{a}$ distribution (see S5 for details). From the original set of 20 climate models, five were selected: cccma-cgcm3-1, cccma-cgcm3-1-t63, mpi-echam5, ukmo-hadcm3, and ukmo-hadgem1 (Table 2, see Figure in Appendix S5).

These climate models were forced with a middle range emissions scenario (SRES A1B, IPCC, 2000). This scenario assumes a future socio-economic development depending on fossil and non-fossil energy sources in balanced proportions. Under this scenario, the $\mathrm{CO}_{2}$ level doubles by 2100, from 360 ppm to 720 ppm.

To generate stochastic $S I C_{a}$ forecasts, we first obtain output for $S I C_{a}$ in each of the four seasons. From this output, we compute smoothed means $\overline{\mathbf{x}}(t)$ and smoothed covariance matrices $\overline{\mathbf{C}}(t)$, using a Gaussian kernel smoother with smoothing parameter $h=2$ (Appendix S5). We then generate stochastic $S I C_{a}$ vectors by drawing $\mathbf{x}(t)$ as an iid sample from a normal distribution with mean $\overline{\mathbf{x}}(t)$ and covariance matrix $\overline{\mathbf{C}}(t)$.

All five GCMs agree that the smoothed mean $S I C_{a}$ will decline by 2100 , but the rate of decline varies between climate models and seasons (Figure 7). For example, $S I C_{a}$ during the laying season is forecast to decline only by $9 \%$ relative to present for the model ukmohadcm3, while $S I C_{a}$ during the non-breeding season will decline by $71 \%$ according to the model cccma-cgm3-t63. There is no clear pattern of change in the smoothed variance (Figure 8), but a high variability over time and between models seems likely. Some models predict a decline in the smoothed variance by the end of the century (e.g. cccma-cgm3-t63 during the non-breeding season), while other an increase (e.g. ukmo-hadcm3 during the laying season). 


\section{Stochastic population projections}

We used each stochastic $S I C_{a}$ forecast to generate a sequence of demographic rates from 2010 to 2100 (Appendix S6). These rates were used to project the population using as an initial population vector the average equilibrium population structure from all the GCMs in 2010. To evaluate uncertainties in climate, we used 200 stochastic forecasts from each of the five GCMs. To evaluate uncertainties in demography, we use the parametric bootstrap approach to generate a sample of 200 simulations for each sea ice forecast. Thus, we project 40,000 population trajectories for each GCM, for a total of 200,000 population trajectories.

The population projections exhibit considerable variability (Figure 9). Some projections produce dramatic declines in the number of breeding pairs (e.g., projections from cccmacgcm3-1), while a few produce large increases (e.g., ukmo-hadcm3). The median of the 40,000 trajectories from each GCM has a unique pattern (Figure 9 and 10). Some increase gradually (e.g., mpi-echam 5), while some decline gradually (e.g., ukmo-hadgem1). Some remain stable for a while and then decline (e.g., cccma-cgcm3-1-t63). For each GCM, however, there exists a year beyond which the median projection declines; this tipping year may be late (e.g., 2089 for ukmo-hadcm3) or early (e.g., 2038 cccma-cgcm3-1-t63). By the end of the century, the medians of all GCMs except ukmo-hadcm3 project that the number of breeding pairs will decline compared to the minimum number over the last six decades.

The median over the entire set of simulations declines to 575 breeding pairs by 2100 . Over this set of simulations, the probability of a decline by $90 \%$ or more by 2100 is 0.43 (Table 3). By 2100, the probability of a decline below the maximum number of breeding pairs ever observed in Terre Adélie since 1962 is 93\% (Table 4). Therefore, only 7\% of population trajectories included in this set are projected to increase by 2100 .

\section{Discussion}

If the climate during the rest of this century follows the patterns forecast by the GCMs examined here, the emperor penguin population at Terre Adélie will respond by declining 
towards extinction. Our median projection shows a decline in the number of breeding pairs by $81 \%$ over this period, and a good chance (43\%) of a more severe decline of $90 \%$ or more. By the end of our projection, the population is continuing to decline, regardless of which GCM is used to forecast future sea ice conditions. The range of uncertainty associated with this result might change the details, but not the overall biological conclusions.

We arrived at this conclusion by tracing the effects of sea ice concentration from the level of the individual to the level of the population. First we measured the response of the vital rates to sea ice conditions, then incorporated those responses into a demographic model to calculate the population growth, and finally we coupled the demographic model to forecasts of sea ice conditions produced by an ensemble of GCMs.

\section{Effects of sea ice on the vital rates}

The vital rates consist of male and female adult survival, the probability of returning to the colony to breed, and breeding success. Previous studies have shown that breeding success and adult survival have the biggest impacts on population growth rate, and that return probability has only a small effect (Jenouvrier et al., 2005a, 2009a).

We found that breeding success declines with increasing values of $S I C_{a}$ during the rearing season. Years with high concentration sea ice may require longer foraging trips, reducing the provisioning of chicks and thus breeding success (Massom et al., 2009; Wienecke \& Robertson, 1997). Not all the variability in breeding success is explained by $S I C_{a}$ during the rearing season, probably because it is affected by many factors; e.g., prolonged blizzards and colder temperatures may increase chick mortality (Jouventin, 1975), and premature ice break-out may cause massive fledging failures (Budd, 1962). We include this unexplained variability in our sea ice-dependent demographic model.

Sea ice affects adult survival in several ways, more complicated than the linear relationships assumed in previous studies (Barbraud \& Weimerskirch, 2001; Jenouvrier et al., 2005a). The effect of annual $S I C_{a}$ on adult survival is greater than the effect of seasonal difference in $S I C_{a}$. Adult survival is maximized at positive annual SIC anomalies $\simeq 2$ and declines 
otherwise. The maximum annual survival is higher for females than males because males are likely more constrained energetically due to their long fasting period (Jenouvrier et al., 2005a). Males incubate the egg, and thus fast for four consecutive months, whereas females return to open water to forage after a two months fasting period. The response of survival to sea ice can be explained by several non-exclusive mechanisms affecting sea ice habitat and constraining the energy expenditure of emperor penguins. During years of concentrated sea ice, foraging trips may be longer, resulting in higher energetic costs (Wienecke \& Robertson, 1997; Massom et al., 2009). On the other hand, during years of concentrated sea ice, food resources may be higher (Barbraud \& Weimerskirch, 2001) and/ or predation lower (Ainley et al., 2007). Seasonal difference in $S I C_{a}$ affects the survival difference between females and males, suggesting that the sexes respond differently to $S I C_{a}$ during the different seasons. Although the mechanisms remain unclear, this difference is probably linked to contrasted energetic costs during breeding.

Male-female survival differences affect the population structure and growth, directly through mortality or indirectly by limiting the availability of mates, and thus reproduction, because penguins are monogamous (Jenouvrier et al., 2010).

\section{Effect of sea ice on population growth rate}

Population growth rate provides a measure of the quality of the environment, in terms of the fitness of a population occupying that environment (Caswell, 2001). The largest effect of sea ice on the population growth of the emperor penguin is due to the annual $S I C_{a}$; the effects of seasonal differences are smaller, but still appreciable.

The deterministic growth rate $\lambda$ is maximized at intermediate values of annual $S I C_{a}$. The maximum occurs at a value of $S I C_{a} \approx 0$ (depending slightly on the value of the seasonal difference in $S I C_{a}$ ). The optimum is relatively broad, as shown by the wide range of annual $S I C_{a}$ values enclosed by the contours for $\log \lambda=0$ in Figure 5 . This implies that changes in sea ice conditions have little effect on $\lambda$ until annual $S I C_{a}$ becomes quite positive or negative. This intermediate optimum is expected because neither complete absence of sea 
ice, nor heavy and persistent sea ice (i.e. no access to resources through polynya) would provide satisfactory conditions for the emperor penguin (Ainley et al., 2010).

A complicated interaction exists between annual $S I C_{a}$ and seasonal differences in $S I C_{a}$. When annual $S I C_{a}$ is lower than -0.8 , the effect of seasonal differences is positive (i.e., the emperor penguin performs better when sea ice concentration is higher in breeding season and lower in non-breeding season). But, when annual $S I C_{a}$ is higher than -0.3 , the effect of seasonal differences is negative; the emperor penguin does better when the seasonal pattern is the opposite.

The emperor penguin population is more sensitive to negative than to positive annual sea ice anomalies; i.e., $\lambda$ decreases from its optimal value faster in the negative than the positive direction (Figure 5). This is well illustrated by the dramatic $50 \%$ population decline in the late 1970s in Terre Adélie, which coincided with several years of the lowest sea ice extent ever recorded during the last 40 years (Jenouvrier et al., 2005c). Other colonies have disappeared in regions with high temperature and low sea ice duration (Fretwell \& Trathan, 2009; Trathan et al., 2011). The Dion Islets colony along the west coast of the Antarctic Peninsula (67 52S, 68 43W) declined from 250 breeding pairs in the 1970s to 20 pairs in 1999, and was extinct by 2009 (Ainley et al., 2010; Trathan et al., 2011). This extinction coincided with a decline in sea ice duration, resulting from a warming of the west coast of the Antarctic Peninsula at an unprecedented rate (Vaughan et al., 2001).

The stochastic population growth rate $\log \lambda_{s}$ shows a similar response to mean annual $S I C_{a}$, with a broad maximum when the mean annual $S I C_{a} \approx 0$. The effect of mean seasonal difference in $S I C_{a}$ is smaller and, as in the deterministic case, is negative when evaluated at a mean annual value of $S I C_{a}=0$ (Figure 6).

The effects of the variance in annual $S I C_{a}$ depend on the value of the mean annual $S I C_{a}$, as shown in Figure 6a. Within the range of approximately $-3 \leq S I C_{a} \leq 4$, the effect of variance is negative, and $\log \lambda_{s}$ is maximized when the variance is zero. But for mean annual $S I C_{a}$ outside this range, the effect of variance on $\log \lambda_{s}$ is positive. 
It is well known that, all else being equal, temporal variance in the vital rates reduces the stochastic growth rate; covariances among vital rates and temporal autocorrelation can reverse this conclusion (Caswell, 2001; Lewontin \& Cohen, 1969; Tuljapurkar, 1990). However, this conclusion does not translate directly to the effect of variance in environmental factors. Because the vital rates are a nonlinear function of sea ice, and the two-sex model is itself nonlinear, the effect of environmental (i.e., $S I C_{a}$ ) variance on population growth may be either positive or negative (e.g., Koons et al. (2009)).

\section{Effect of future sea ice change}

Our best projection of the future growth of the Terre Adélie emperor penguin population, under the impact of climate change, is a decline by the year 2100 from approximately 3000 breeding pairs to 575 breeding pairs, a decline of $81 \%$, or an average rate of change of -0.018 per year. This projection is the median of a large set of simulations that incorporate as many sources of uncertainty as possible.

The median is a smoothed pattern based on 200,000 population trajectories. Each of those trajectories fluctuates, with increases, decreases, and periods of relative stability, until it reaches a tipping year, after which it declines. The tipping year varies among trajectories, depending on the forecasted sea ice, the responses of the vital rates, and the impact of sex-specific adult survival on the demography. The decline in the median of the 200000 population trajectories accelerates after 2040 because more of the population trajectories are likely to have reached their tipping year as time goes on.

These population projections required three steps.

1. We had to extract the biologically relevant GCM outputs, in our case the seasonal $S I C_{a}$ values, on appropriate spatial scales. The choice of spatial scales is an important issue in studies of climate change using GCMs, which project sea ice variables, such as concentration, thickness (SIT) and extent, over a greater spatial scale than the scale of emperor penguin habitat requirements. For emperor penguins, the size of polynyas (Ainley et al., 2005), sea ice thickness, area of fast ice (Massom et al., 2009), the timing 
of ice breakup and formation are meaningful variables with respect to the life cycle (see review in Ainley et al. (2010)) but are measurable only at small spatial scales. By focusing on SIC we hope to reduce the number of correlated covariates (Grosbois et al., 2008). At large spatial scales, SIC is strongly correlated to SIT and open water area, and thus SIC is a good measure of the sea ice environment experienced by penguins. Further climate research will be required to downscale the sea ice projections of GCMs in Antarctica both spatially (e.g. RCMs) and temporally (e.g. daily data to calculate the timing of ice breakup) as climate model output is typically saved and available with a monthly resolution.

2. We had to model temporal variance in $S I C_{a}$ from the GCM output. Ideally, this would be obtained from multiple stochastic realizations of each GCM, but such output does not exist. Thus, we obtained smoothed temporal means, variances, and covariances from the output, and used those to parameterize variability in $S I C_{a}$ at each time step. The forecasts of smoothed temporal means and variances of $S I C_{a}$ differ strongly among climate models. Differences are present in numerous aspects of the climate models, including their physical parameterizations and spatial resolution. As such, it is difficult to attribute differences in the projected sea ice change to a single factor. However, as noted by Lefebvre \& Goosse (2008), numerous mechanisms do appear to play a role. These include the influence of local simulated climate conditions at the end of the 20th century, aspects of the atmospheric Southern Annular Mode response (e.g. Fyfe \& O.A. (2006)), and changes in the simulated response of the Southern Ocean circulation. While our climate model selection process does reduce the uncertainty associated with the first of these, differences in simulated feedbacks and climate response will still play an important role. This points to a further need for climate model development and enhancement.

3. It was critical to incorporate uncertainty in our projections; a projection of an $81 \%$ decline without an associated range of uncertainty would be useless. Our uncertainty 
analysis included components from the demographic parameters and their response to sea ice conditions, including both sampling error and the uncertainty due to model selection. We also included uncertainty in future sea ice conditions by using an ensemble of GCMs, chosen on the basis of their agreement with past sea ice observations. Figure 9 shows the variation among the models in their forecasts of $S I C_{a}$, and Figure 10 provides a powerful graphical summary of the resulting uncertainty in population forecasts. Models cccma-cgcm3-1 and cccma-cgcm3-1-t63 predict the most rapid declines, with the population reduced to 20 or 11 breeding pairs, respectively, by 2100 . In contrast, model ukmo-hadcm-3 makes more optimistic sea ice forecasts, and projects an increase in the population by 2100 .

Stochasticity and uncertainty naturally lead to significant variation among projections of future emperor penguin population growth. Among the 200,000 population trajectories, there are some examples in which the population does not decline, or even increaes, but the central tendency is an unambiguous and serious population decline of $81 \%$. More important, the probability distributions of projected population size in 2100 show that declines are far more likely than increases or stability. In addition, the median population trends predicted by all five GCMs during the last decade (2090-2100), even the outlier ukmo-hadcm3, are negative. Thus the difference between climate models, i.e. uncertainty in the sea ice forecasts, affects the timing of the population median decline, but not whether that median decline occurs or not (Figure 10).

The conclusion that the emperor penguin population will decline dramatically by the end of this century raises the issue of possible adaptation. Emperor penguins might adapt to the new sea ice conditions or, more likely, disperse to locations where sea ice conditions are more favorable (see review Ainley et al. (2010); Forcada \& Trathan (2009) and discussion in Trathan et al. (2011); Jenouvrier et al. (2009b)). Future studies should quantify potential refuges for the species (but see Ainley et al. (2010)) and consider potential evolutionary responses. Thus, we encourage ecologists to collect information on phenotypic traits and 
their heritability.

The median projection of 575 breeding pairs in 2100 is close to the projection of $\approx 400$ breeding pairs obtained previously using a simpler modelling approach (Jenouvrier et al. 2009). Consistencies among population projections from different demographic and climate models increase confidence in the assessment of the impact of climate change.

\section{Methodological notes}

Our approach to predicting and understanding the effects of climate change requires the measurement of effects of climate on individuals, the integration of those effects into a demographic model, and the connection of the demographic model to climate forecasts. Aspects of this approach have been applied to some other species, including seabirds (Barbraud et al., 2010; Wolf et al., 2010), polar bears (Hunter et al., 2010), the oystercatcher (van de Pol et al., 2010), and our previous analysis of the emperor penguin (Jenouvrier et al., 2009b). In this study, we have gone beyond these studies in several ways.

Because of the length and quality of the emperor penguin data set, in this analysis we were able to identify detailed effects of sea ice on a seasonal basis through the breeding cycle, and to include, for the first time, the nonlinear effects of sex-specific mortality patterns using a two-sex demographic model. In the case of the emperor penguin, the extreme conditions of its breeding cycle make the presence of both parents essential for successful reproduction. Hence, sex-specific climate effects are particularly important (cf. Jenouvrier et al. (2010)). In general, however, we expect that even in less dramatic conditions, the different roles of the sexes may often cause climate change impacts to differ between males and females.

Because of the ability to extract relevant sea ice output from the GCMs, we were able to include both stochasticity and uncertainty, and to draw conclusions about the projected fate of the Terre Adélie emperor penguin population even in the face of that uncertainty. Because climate models predict that both the mean and variability of climate will change (Solomon et al., 2007), it is important to include these stochastic effects.

Two factors not included in our models deserve mention: density dependence and demo- 
graphic stochasticity. It is almost reflexive to ask about density dependence in an analysis such as ours. This is a result of exposure to simple scalar population models. In a stagestructured population, especially in a highly seasonal environment, and in which different stages occupy vastly different environments during the course of the year, population growth is never likely to be a function of something as simple as "density." In the case of the emperor penguin, it is a priori unlikely that density has strong effects, and in Appendix 1, 3 we describe our analysis of the effects of the number of breeding pairs on the vital rates; we found no support for these effects.

Demographic stochasticity refers to fluctuations arising from the random outcome of probabilities of survival and reproduction, applied to individuals in a population. It can be analyzed using multi-type branching process models (Chapter 15 of Caswell 2011). Demogrpaphic stochasticity is unimportant in large populations, but reduces the stochastic growth rate and increases extinction probability in small populations. As the emperor penguin population declines, demographic stochasticity will, at some point, become important. In general, however, this requires population sizes of only a few tens to a hundred individuals. To the extent that demographic stochasticity becomes important at the end of our simulations, our projections overestimate the persistence of the population.

We encourage further collaborations between ecologists and climatologists. The development of data archive resources such as those provided by the National Snow and Ice Data Center (NSIDC) and the Program for Climate Model Diagnosis and Intercomparison (PCMDI) (Meehl et al., 2007) have allowed unprecedented access to observed and modeled climate data. We believe that the participation of climatologists is critical for selecting the most appropriate set of climate models, emissions scenarios, climate variables, and the use of climate ensembles versus single climate outputs.

An important future step will be to incorporate evolutionary responses. A theoretical framework to link life history traits and population dynamics (eco-evolutionary dynamics) is emerging (Hoffmann \& Sgrò, 2011; Pelletier et al., 2009; Kinnison \& Hairston, 2007; Reed 
et al., 2010), and studies of the potential evolutionary responses to climate change and their population consequences have recently been initiated (Jenouvrier \& Visser (2011); Reed et al. (2011)). We believe that the way climatologists have approached the problem, using coupled climate system models in which climate systems components (e.g. ocean, atmosphere, sea ice, land surface, ice sheets, biogeochemistry and more) are gradually connected, can provide a valuable example to ecologists toward an integrative climate- eco-evolutionary framework.

\section{Acknowledgments}

The penguin data come from a long-term study supported by Expéditions Polaires Françaises, by Institut Paul Emile Victor (Programme IPEV 109), and by Terres Australes et Antarctiques Françaises. We acknowledge the efforts of all the wintering fieldworkers involved in the long-term monitoring programs in Terre Adélie since 1963, and thank Dominique Besson and Karine Delord for the management of the database. We acknowledge the modeling groups, the Program for Climate Model Diagnosis and Intercomparison (PCMDI) and the WCRP's Working Group on Coupled Modelling (WGCM) for their roles in making available the WCRP CMIP3 multi-model dataset. Support of this dataset is provided by the Office of Science, U.S. Department of Energy. HW, CB and SJ acknowledge support from the REMIGE (Behavioural and demographic REsponses of Indian Ocean Marine top predators to Global Environmental changes) program funded by ANR (Agence Nationale de la Recherche) Biodiversité (ANR Biodiv 011). This work was carried out during the tenure of Marie-Curie and CIRES Visiting fellowships by S.J. MH acknowledges support through the National Science Foundation. HC acknowledges support from NSF Grant DEB-0816514, from the WHOI Arctic Research Initiative, and from the Alexander von Humboldt Foundation. The authors thank the two anonymous reviewers and David Koons for their valuable comments and suggestions on previous manuscript version. 


\section{References}

Adahl E, Lundberg P, Jonzen N (2006) From climate change to population change: the need to consider annual life cycles. Global Change Biology, 12, 1627-1633.

Ainley D, Ballard G, Ackley S, et al. (2007) Paradgim lost, or is top-down forcing no longer significant in the antarctic ecosystem? Antarctic Science.

Ainley D, Clarke E, Arrigo K, Fraser B, Kato A, Barton K, Wilson P (2005) Decadal-scale changes in the climate and biota of the pacific sector of the southern ocean, 1950s to the 1990s. Antarctic Science, 17, 171-182.

Ainley D, Russell J, Jenouvrier S, Woehler E, Lyver P, Fraser W, Kooyman G (2010) Antarctic penguin response to habitat change as earths troposphere reaches 2 degrees above preindustrial levels. Ecological Monographs, 80, 49-66.

Akaike H (1974) A new look at the statistical model identification. IEEE Transactions on Automatic Control, 19 (6):, 716723.

Atkinson A, Siegel A, Pakhomov E, Rothery P (2004) Long-term decline in krill stock and increase in salps within the southern ocean. Nature, 432, 100-103.

Atkinson A, Siegel V, Pakhomov E, et al. (2008) Oceanic circumpolar habitats of antarctic krill. Marine Ecology Progress Series, 362, 1-23.

Ballerini T, Tavecchia G, Olmastroni S, Pezzo F, Focardi S (2009) Nonlinear effects of winter sea ice on the survival probabilities of adélie penguins. Oecologia, 161, 253-265.

Barbraud C, Cotte C (2008) Short note: Paradigms need hypothesis testing: no evidence for top-down forcing on adélie and emperor penguin populations. Antarctic Science, 20, 2 . 
Barbraud C, Rivalan P, Inchausti P, Nevoux M, Rolland V, Weimerskirch H (2010) Contrasted demographic responses facing future climate change in southern ocean seabirds. Journal of Animal Ecology, 80, 89-100.

Barbraud C, Weimerskirch H (2001) Emperor penguins and climate change. Nature, 411, $183-186$.

Both C, Bouwhuis S, Lessells CM, Visser ME (2006) Climate change and population declines in a long-distance migratory bird. Nature, 441, 81-83.

Brierley A, Fernandes P, Brandon M, et al. (2002) Antarctic krill under sea ice : Elevated abundance in a narrow band just south of ice edge. Science, 295, 1890-1892.

Budd GM (1962) opulation studies in rookeries of the emperor penguin aptenodytes forsteri. Proceedings of the Zoological Society of London, 139, 365.

Burnham K, Anderson D (2002) Model Selection and Multimodel Inference: A Practical Information-theoretic Approach. Springer.

Caswell H (2001) Matrix population models, vol. Second. Sinauer, Sunderland, Massachusetts.

Cavalieri D, Parkinson C, Gloersen P, Zwally HJ (1996) Sea ice concentrations from nimbus7 smmr and dmsp ssm/i passive microwave data. Boulder, Colorado USA: National Snow and Ice Data Center. Digital media.

Cherel Y (2008) Isotopic niches of emperor and adélie penguins in adélie land, antarctica. Marine Biology, 154, 813-821.

Cherel Y, Kooyman G (1998) Food of emperor penguin (Aptenodytes forsteri) in the western ross sea, antarctica.hu. Marine Biology, 130, 335-344. 
Choquet R, Reboulet A, Pradel R, Giménez O (2004) M-surge: new software specifically designed for multistate capture-recapture models. Animal Biodiversity and Conservation, 27, 207-215.

Croxall J, Trathan P, Murphy E (2002) Environmental change and antarctic seabirds populations. Science, 297, 1510-1514.

Forcada J, Trathan PN (2009) Penguin responses to climate change in the southern ocean. Global Change Biology, 15, 1618-1630.

Fraser W, Hoffmann E (2003) A predator's perspective on causal links between climate change, physical forcing, and ecosystem response. Marine Ecology Progress Series, 265, $1-15$.

Fretwell PT, Trathan PN (2009) Penguins from space: faecal stains reveal the location of emperor penguin colonies. Global Ecology and Biogeography, 18, 543-552.

Fyfe J, OA S (2006) Simulated changes in the extratropical southern hemisphere winds and currents. Geophysical Research Letters, 33, L06701.

Grosbois V, Gimenez O, Gaillard J, et al. (2008) Assessing the impact of climate variation on survival in vertebrate populations. Biological Reviews, 83, 357-399.

Herrando-Pérez S, Delean S, Brook B, Bradshaw CJA (2012) Decoupling of component and ensemble density feedbacks in birds and mammals. Ecology.

Hoffmann AA, Sgrò CM (2011) Climate change and evolutionary adaptation. Nature, 470, $479-485$.

Hughes L (2000) Biological consequences of global warming: is the signal already apparent? Trends in Ecology and Evolution, 15, 56-61. 
Hunter C, Caswell H, Runge M, Regehr E, Amstrup S, Stirling I (2010) Climate change threatens polar bear populations: a stochastic demographic analysis. Ecology, 91, 28832897.

Jenouvrier S, Barbraud C, Weimerskirch H (2003) Effects of climate variability on the temporal population dynamics of southern fulmars. Journal of Animal Ecology, 72, 576-587.

Jenouvrier S, Barbraud C, Weimerskirch H (2005a) Long-term contrasted responses to climate of two antarctic seabirds species. Ecology, 86, 2889-2903.

Jenouvrier S, Barbraud C, Weimerskirch H (2005b) Sea ice affects the population dynamics of adélie penguins in terre adélie. Polar Biology, 29, 413-423.

Jenouvrier S, Barbraud C, Weimerskirch Hand Caswell H (2009a) Limitation of population recovery: a stochastic approach to the case of the emperor penguin. Oikos, 118, 12921298.

Jenouvrier S, Caswell H, Barbraud C, Holland M, Stroeve J, Weimerskirch H (2009b) Demographic models and ipcc climate projections predict the decline of an emperor penguin population. Proceedings of the National Academy of Sciences, 106, 1844-1847.

Jenouvrier S, Caswell H, Barbraud C, Weimerskirch H (2010) Mating behavior, population growth, and the operational sex ratio: A periodic two-sex model approach. The American naturalist, 175, 739-752.

Jenouvrier S, Visser M (2011) Climate change, phenological shifts, eco-evolutionary responses and population viability: toward a unifying predictive approach. Journal of Biometeorology, 458, 1-15.

Jenouvrier S, Weimerskirch H, Barbraud C, Park YH, Cazelles B (2005c) Evidence of a shift in cyclicity of antarctic seabirds dynamics link to climate. Proceedings of the Royal Society of London.B., 272, 887-895. 
Jouventin P (1975) The Biology of Penguins., chap. Mortality parameters in emperor penguins Aptenodytes forsteri., p. 435446. Macmillan Press, London.

Kinnison M, Hairston N (2007) Eco-evolutionary conservation biology: contemporary evolution and the dynamics of persistence. Functional Ecology, 21, 444-454.

Kirkwood R, Robertson G (1997) The foraging ecology of female emperor penguins in winter. Ecological Monograph, 67, 155-176.

Koons DN, Pavard S, Baudisch A, Metcalf CJE (2009) Is life-history buffering or lability adaptive in stochastic environments? Oikos, 118, 972-980.

Lebreton J, Burnham K, Clobert J, Anderson D (1992) Modeling survival and testing biological hypotheses using marked animals: a unified approach with case studies. Ecological Monographs, 62, 67-118.

Lebreton JD, Nichols JD, Barker RJ, Pradel R, Spendelow JA (2009) Modeling individual animal histories with multistate capture-recapture models. Advances in Ecological Research, 41, 87-173.

Lefebvre W, Goosse H (2008) Analysis of the projected regional sea-ice changes in the southern ocean during the twenty-first century. Climate dynamics, 30, 59-76.

Lewontin RC, Cohen D (1969) On population growth in a randomly varying environment. Proceedings of the National Academy of Sciences, pp. 1056-1060.

Loeb V, Siegel V, Holm-Hansen O, Hewitt R, Fraser W, Trivelpiece S (1997) Effects of sea-ice extend and krill or salp dominance on the antarctic food web. Nature, 387, 897-900.

Massom R, Hill K, Barbraud C, Adams N, Ancel A, Emmerson L, Pook M (2009) Fast ice distribution in adélie land, east antarctica: interannual variability and implications for emperor penguins aptenodytes forsteri. Marine Ecology Progress Series, 374, 243-257. 
McCarty J (2001) Ecological consequences of recent climate change. Conservation Biology, 15, 320-331.

Meehl GA, Covey C, Delworth T, et al. (2007) The wcrp cmip3 multi-model dataset: A new era in climate change research. Bulletin of the American Meteorological Society, $\mathbf{8 8}$, 1383-1394.

Moller AP, Rubolini D, Lehikoinen E (2008) Populations of migratory bird species that did not show a phenological response to climate change are declining. Proceedings of the National Academy of Sciences, 105, 16195-16200.

Nicol S, Pauly T, Bindoff N, et al. (2000) Ocean circulation off east antarctica affects ecosystem structure and sea-ice extent. Nature, 406, 504-507.

Parmesan C (2006) Ecological and evolutionary responses to recent climate change. Annual Review of Ecology, Evolution, and Systematics, 37, 637-669.

Parmesan C, Yohe G (2003) A globally coherent fingerprint of climate change impacts across natural systems. Nature, 421, 37-42.

Pelletier F, Garant D, Hendry A (2009) Eco-evolutionary dynamics. Philosophical Transactions $B, \mathbf{3 6 4}, 1483-1489$.

Pitman RL, Durban JW (2010) Killer whale predation on penguins in antarctica. Polar Biology, 33, 1589-1594.

Prevost J (1961) Ecologie du manchot empereur. E.P.F., 222.

Quetin L, Ross R, Fritsen C, Vernet M (2007) Ecological responses of antarctic krill to environmental variability: can we predict the future? Antarctic Science, 19, 253-266.

Reed T, Schindler D, Waples RS (2010) Interacting effects of phenotypic plasticity and evolution on population persistence in a changing climate. Conservation Biology, 25, $56-63$. 
Reed TE, Schindler DE, Hague MJ, Patterson DA, Meir E, Waples RS, Hinch SG (2011) Time to evolve? potential evolutionary responses of fraser river sockeye salmon to climate change and effects on persistence. Plos One, 6, e20380.

Regehr EV, Hunter CM, Caswell H, Amstrup SC, Stirling I (2010) Survival and breeding of polar bears in the southern beaufort sea in relation to sea ice. Journal of Animal Ecology, 79, $117-127$.

Saraux C, Bohec CL, Durant JM, et al. (2011) Reliability of flipper-banded penguins as indicators of climate change. Nature, 469, 203-206.

Solomon S, D Qin MM, Chen Z, Marquis M, Averyt K, Tignor M, Miller H (2007) Contribution of Working Group I to the Fourth Assessment Report of the Intergovernmental Panel on Climate Change, 200\%. Cambridge University Press, Cambridge, United Kingdom and New York, NY, USA.

Stenseth N, Mysterud A, Ottersen G, Hurell J, Chan K, Lima M (2002) Ecological effects of climate fluctuations. Science, 297, 1292-1295.

Stock CA, Alexander MA, Bond NA, et al. (2011) On the use of ipcc-class models to assess the impact of climate on living marine resources. Progress in Oceanography, 88, 1-27.

Thomas D, Dieckmann G (2003) Sea ice: an introduction to its physics, chemistry, biology and geology. Oxford: Blackwell Science, 240-266 pp.

Trathan PN, Fretwell PT, Stonehouse B (2011) First recorded loss of an emperor penguin colony in the recent period of antarctic regional warming: Implications for other colonies. Plos One, 6, e14738.

Tuljapurkar S (1990) Delayed reproduction and fitness in variable environments. Proceedings of the National Academy of Sciences, 87, 1139. 
van de Pol M, Vindenes Y, Saether BE, Engen S, Ens BJ, Oosterbeek K, Tinbergen JM (2010) Effects of climate change and variability on population dynamics in a long-lived shorebird. Ecology, 91, 1192-1204.

Vaughan D, Marshall G, Connolley W, King J, Mulvaney R (2001) Devil in the detail. Science, 293, 1777-1779.

Visser M, van Noordwijk AJand Tinbergen J, Lessells C (1998) Warmer springs lead to mistimed reproduction in great tits (Parus major). Proceedings of the Royal Society B: Biological Sciences, 265, 18671870.

Vitousek P (1994) Beyond global warming: Ecology and global change. Ecology, 75, 18611876.

Walther G, Post E, Convey P, et al. (2002) Ecological responses to recent climate change. Nature, 416, 389-395.

Wiedenmann J, Cresswell K, Mangel M (2009) Connecting recruitment of antarctic krill and sea ice. Limnology and Oceanography, 54, 799-811.

Wienecke BC, Robertson G (1997) Foraging space of emperor penguins Aptenodytes forsteri in antarctic shelf water in winter. Marine Ecology Progress Series, 159.

Wolf SG, Snyder MA, Sydeman WJ, Doak DF, Croll DA (2010) Predicting population consequences of ocean climate change for an ecosystem sentinel, the seabird cassin's auklet. Global Change Biology, 16, 1923-1935.

Zimmer I, Wilson R, Gilbert C, Beaulieu M, Ancel a, Plotz J (2008) Foraging movements of emperor penguins at pointe géologie, antarctica. Polar Biology, 31, 229-243. 


\section{Supporting Information legends}

Appendix 1 discusses the effect of density dependence.

Appendix 2 describes the selection of sea ice variables and details the principal component analysis.

Appendix 3 details vital rates estimation (model selection, estimates and 95\% confidence intervals) for: (2.1) breeding success, (2.2) adult survival, and (2.3) probabilities of return to the colony.

Appendix 4 details the effect of sea ice variability on demography. Figures show the distributions of annual $S I C_{a}$, breeding success, and male and female adult survival for females and males, along with the resulting distribution of the deterministic growth rate $\lambda$. This deterministic rate can be thought of as approximating the growth of the population between time $t$ and $t+1$, although this is not always true (see Appendix).

Appendix 5 shows the sea ice projected by each climate model for each season of the penguin's life cycle and the climate selection procedure (section 4.1). It also details our novel approach to obtain stochastic sea ice forecasts from single climate output (section $4.2)$.

Appendix 6 describes the projections of vital rates in the future and shows that the range of variation in the forecast vital rates from 2010 to 2100 is plausible. 
${ }_{832} 8$ Tables 
Table 1: Results of model selection for annual adult survival, including linear or quadratic effects of $S I C_{a}$ at each of four seasons: non-breeding, laying, incubating and rearing. The first and second columns give the effects included in the model for females and males respectively. The number of parameters in the model is $K, \triangle A I C$ is the difference in Akaikes information criterion (AIC) between each model and the model with the smallest AIC (i.e. best supported by the data). AIC weights represent the relative likelihood of a model and are used to create the averaged model; only models for which the cumulative sum of AIC weights is 0.98 are included.

\begin{tabular}{|c|c|c|c|c|}
\hline Effect for females & Effect for males & $K$ & $\triangle A I C$ & AIC weight \\
\hline incubating & incubating & 135 & 0.00 & 0.14 \\
\hline rearing $^{2}$ & rearing $^{2}$ & 137 & 0.78 & 0.09 \\
\hline rearing $^{2}$ & incubating & 136 & 1.17 & 0.08 \\
\hline incubating & incubating $^{2}$ & 136 & 1.25 & 0.07 \\
\hline rearing $^{2}$ & time-invariant & 135 & 1.59 & 0.06 \\
\hline rearing $^{2}$ & incubating $^{2}$ & 137 & 2.11 & 0.05 \\
\hline rearing & time-invariant & 134 & 2.22 & 0.04 \\
\hline rearing & incubating & 135 & 2.46 & 0.04 \\
\hline rearing & laying & 135 & 2.93 & 0.03 \\
\hline rearing $^{2}$ & rearing & 136 & 3.53 & 0.02 \\
\hline time-invariant & time-invariant & 133 & 3.63 & 0.02 \\
\hline rearing & rearing $^{2}$ & 136 & 3.71 & 0.02 \\
\hline incubating & time-invariant & 134 & 3.73 & 0.02 \\
\hline rearing & non breeding & 135 & 3.94 & 0.02 \\
\hline rearing $^{2}$ & laying & 136 & 4.00 & 0.02 \\
\hline rearing & rearing & 135 & 4.12 & 0.02 \\
\hline incubating & rearing $^{2}$ & 136 & 4.13 & 0.02 \\
\hline incubating & rearing & 135 & 4.14 & 0.02 \\
\hline rearing & incubating $^{2}$ & 136 & 4.15 & 0.02 \\
\hline rearing & incubating $^{2}$ & 136 & 4.15 & 0.02 \\
\hline rearing $^{2}$ & non breeding & 136 & 2.97 & 0.03 \\
\hline time-invariant & incubating & 134 & 4.46 & 0.01 \\
\hline laying & incubating & 135 & 4.52 & 0.01 \\
\hline incubating $^{2}$ & incubating $^{2}$ & 137 & 4.62 & 0.01 \\
\hline incubating & laying & 135 & 4.81 & 0.01 \\
\hline time-invariant & non breeding & 134 & 4.99 & 0.01 \\
\hline laying & time-invariant & 134 & 5.25 & 0.01 \\
\hline time-invariant & rearing & 134 & 5.25 & 0.01 \\
\hline incubating & non breeding & 135 & 5.49 & 0.01 \\
\hline incubating & rearing $^{2}$ & 136 & 5.50 & 0.01 \\
\hline laying & incubating $^{2}$ & 136 & 5.75 & 0.01 \\
\hline time-invariant & incubating $^{2}$ & 135 & 5.88 & 0.01 \\
\hline non breeding & time-invariant & 134 & 6.02 & 0.01 \\
\hline incubating $^{2}$ & rearing $^{2}$ & 137 & 6.10 & 0.01 \\
\hline time-invariant & laying & 134 & 6.31 & 0.01 \\
\hline laying & rearing $^{2}$ & 136 & 6.34 & 0.01 \\
\hline
\end{tabular}


Table 2: Selection of General Circulation Models for climate. Each model was evaluated by comparing the statistical properties of its sea ice output to observed data from 1979-2007. Agreement is indicated by an $\mathrm{x}$; the GCMs selected are shown in bold.

\begin{tabular}{|c|c|c|c|c|}
\hline Model & Non-breeding & Laying & Incubating & Rearing \\
\hline \multicolumn{5}{|l|}{ bccr-bcm2-0 } \\
\hline cccma-cgcm3-1 & $\mathbf{x}$ & $\mathbf{x}$ & $\mathbf{x}$ & $\mathbf{x}$ \\
\hline cccma-cgcm3-1-t63 & $\mathrm{x}$ & $\mathbf{x}$ & $\mathrm{x}$ & $\mathbf{x}$ \\
\hline cnrm-cm3 & & & $\mathrm{x}$ & $\mathrm{x}$ \\
\hline csiro-mk3-0 & & & $\mathrm{x}$ & $\mathrm{x}$ \\
\hline gfdl-cm2-0 & & $\mathrm{x}$ & & $\mathrm{x}$ \\
\hline gfdl-cm2-1 & & & $\mathrm{x}$ & \\
\hline giss-aom & $\mathrm{x}$ & & $\mathrm{x}$ & $\mathrm{x}$ \\
\hline \multicolumn{5}{|l|}{ giss-model-e-r } \\
\hline \multicolumn{5}{|l|}{ iap-fgoals1-0-g } \\
\hline \multicolumn{5}{|l|}{ inmcm3-0 } \\
\hline ipsl-cm4 & & & $\mathrm{x}$ & $\mathrm{x}$ \\
\hline miroc3-2-hires & & $\mathrm{x}$ & $\mathrm{x}$ & $\mathrm{x}$ \\
\hline miroc3-2-medres & & $\mathrm{x}$ & $\mathrm{x}$ & $\mathrm{x}$ \\
\hline \multicolumn{5}{|l|}{ miub-echo-g } \\
\hline mpi-echam5 & $\mathbf{x}$ & $\mathbf{x}$ & $\mathbf{x}$ & $\mathbf{x}$ \\
\hline mri-cgcm2-3-2a & $\mathrm{x}$ & & & \\
\hline ncar-ccsm3-0 & $\mathrm{x}$ & $\mathrm{x}$ & & $\mathrm{x}$ \\
\hline ukmo-hadcm3 & $\mathbf{x}$ & $\mathbf{x}$ & $\mathbf{x}$ & $\mathbf{x}$ \\
\hline ukmo-hadgem1 & $\mathrm{x}$ & $\mathrm{x}$ & $\mathbf{x}$ & $\mathbf{x}$ \\
\hline
\end{tabular}


Table 3: Probability that the emperor penguin population in Terre Adélie will decline by more than $90 \%$ from 2010 to 2040, 2060, 2080, and 2100, when sea ice follows the forecasts of each of the five climate models selected.

\begin{tabular}{lcccc}
\hline models & 2040 & 2060 & 2080 & 2100 \\
\hline cccma-cgcm3-1 & 0.0168 & 0.2366 & 0.7625 & 0.9903 \\
cccma-cgcm3-1-t63 & 0 & 0.0205 & 0.6783 & 0.9997 \\
ukmo-hadcm3 & 0 & 0 & 0 & 0.0001 \\
ukmo-hadgem1 & 0 & 0.0001 & 0.0088 & 0.1276 \\
mpi-echam5 & 0 & 0 & 0 & 0.0181 \\
\hline entire set & 0 & 0.0514 & 0.2899 & 0.4272 \\
\hline
\end{tabular}


Table 4: Probability that the emperor penguin population in Terre Adélie will decline below a specific number of breeding pairs (threshold) by 2100, when sea ice follows the forecasts of each of the five climate models selected. Thresholds are based on the minimum and maximum number of observed breeding pairs (Nobs) during specific time periods (3 first columns), or specific numbers (last two columns).

\begin{tabular}{lccccc}
\hline threshold & $\min \left(\right.$ Nobs $\left._{1979-2010}\right)$ & $\max \left(\right.$ Nobs $\left._{1979-2010}\right)$ & $\max \left(N_{\text {obs }} 1962-2010\right)$ & & \\
& 2303 & 3482 & 6236 & 8000 & 10000 \\
\hline cccma-cgcm3-1 & 1.00 & 1.00 & 1.00 & 1.00 & 1.00 \\
cccma-cgcm3-1-t63 & 1.00 & 1.00 & 1.00 & 1.00 & 1.00 \\
ukmo-hadcm3 & 0.21 & 0.43 & 0.77 & 0.87 & 0.93 \\
ukmo-hadgem1 & 0.94 & 0.98 & 1.00 & 1.00 & 1.00 \\
mpi-echam5 & 0.75 & 0.90 & 0.99 & 1.00 & 1.00 \\
\hline entire set & 0.75 & 0.84 & 0.93 & 0.96 & 0.98 \\
\hline
\end{tabular}




\section{Figures}

Figure 1: Observed proportional anomalies in sea ice concentration $\left(S I C_{a}\right)$ relative to the mean from 1979 to 2007, for each of four seasons of the penguin life cycle. The grey line shows $S I C_{a}=0$ and represents the mean SIC from 1979 to 2007.

Figure 2: (a) Seasonal SIC anomalies (color lines, see legend) as a function of the annual $S I C_{a}$, with the seasonal differences in $S I C_{a}$ set to zero. (b) Seasonal SIC anomalies as a function of the seasonal differences in $S I C_{a}$, with the annual $S I C_{a}$ set to zero.

Figure 3: Breeding success as a function of proportional anomalies in SIC during the rearing season. The line is a logistic regression fit to the data points shown.

Figure 4: Annual adult survival as a function of annual $S I C_{a}$ and seasonal differences in $S I C_{a}$. (a c) and (b d) show the survival of females and males respectively. Upper panels (a b) show survival as a function of annual $S I C_{a}$ for negative (=-1, grey line), zero (thick black line), and positive $\left(=+1\right.$, dotted line) values of seasonal differences in $S I C_{a}$. Lower panels (c d) show survival as a function of seasonal differences in $S I C_{a}$ for negative (=-4, grey line), zero (thick black line), and positive $\left(=+4\right.$, dotted line) values of annual $S I C_{a}$.

Figure 5: The deterministic population growth rate $(\log \lambda)$ as a function of annual $S I C_{a}$ and seasonal differences in $S I C_{a}$. The white contours indicate $\log \lambda=0$. The color bar shows the values of $\log \lambda$.

Figure 6: The stochastic population growth rate $\log \lambda_{s}$ as a function of the mean and variance of sea ice variables. (a) $\log \lambda_{s}$ as a function of the mean and variance of annual $S I C_{a}$ with seasonal differences in $S I C_{a}$ set to zero. The black line stands for a zero variance, the grey line for the observed variance, and the dotted line for a variance equal to twice the observed. (b) $\log \lambda_{s}$ as a function of the mean and variance of seasonal differences in $S I C_{a}$, with annual $S I C_{a}$ equal to zero.

Figure 7: (a) Smoothed mean of $S I C_{a}$ during the four seasons, from each of the five selected GCMs (color lines, see legend). The black line shows the observed $S I C_{a}$ from 1979 to 2007 and the red line shows the zero value, i.e. represents averaged SIC from 1979 to 
2007.

Figure 8: Smoothed standard deviation of $S I C_{a}$ during the four seasons, from each of the five selected GCMs (color lines, see legend on figure 7). The red line shows the observed standard deviation. The grey box represents 0.5 and 1.5 times the observed standard deviation; these values were used in selecting climate models (Appendix 4).

Figure 9: Projections of the emperor penguin population based on $S I C_{a}$ forecasts from an ensemble of five GCMs. The black line gives the observed number of breeding pairs from 1979 to 2010. For each GCM, three random population trajectories are shown (thin colored lines), along with the median (thick colored line) and the 95\% envelope (grey area), from 40,000 stochastic simulations. The median and 95\% envelope are also shown from the combined 200,000 simulations for the set of 5 GCMs.

Figure 10: Summary of the projections of the emperor penguin population based on $S I C_{a}$ forecasts from an ensemble of five GCMs. The thick colored lines (see legend on figure 9) give the median and the grey area is the $95 \%$ envelope from the combined 200,000 simulations for the set of 5 GCMs. Also shown are the probability density functions for simulated population size in 2100, for each GCM. 

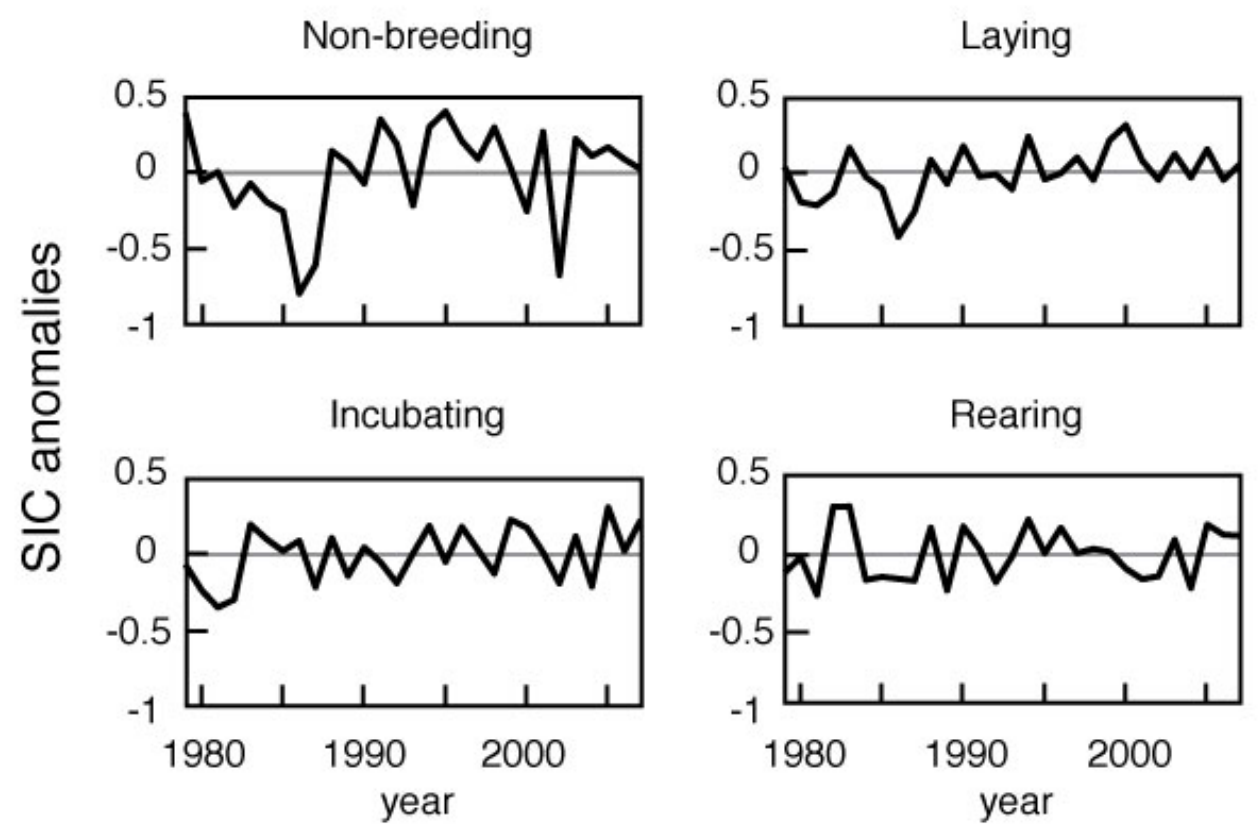

Figure 1: Observed proportional anomalies in sea ice concentration (SIC) relative to the mean from 1979 to 2007, for each of four seasons of the penguin life cycle. The grey line shows $S I C_{a}=0$ and represents the mean SIC from 1979 to 2007. 
(a)

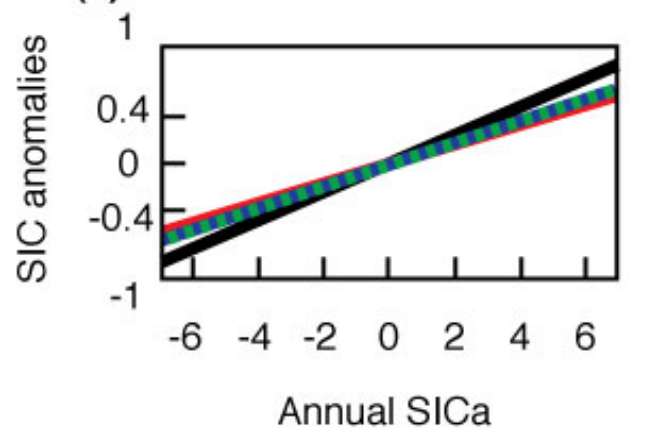

— non-breeding _ _ laying (b)

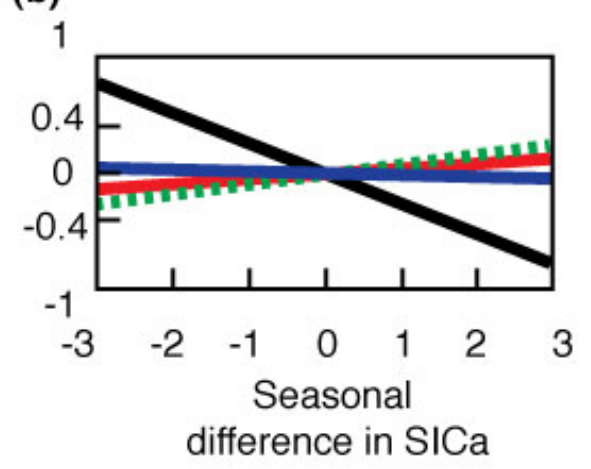

incubating

Figure 2: (a) Seasonal SIC anomalies as a function of the annual $S I C_{a}$, with the seasonal differences in $S I C_{a}$ set to zero. (b) Seasonal SIC anomalies as a function of the seasonal differences in $S I C_{a}$, with the annual $S I C_{a}$ set to zero. 


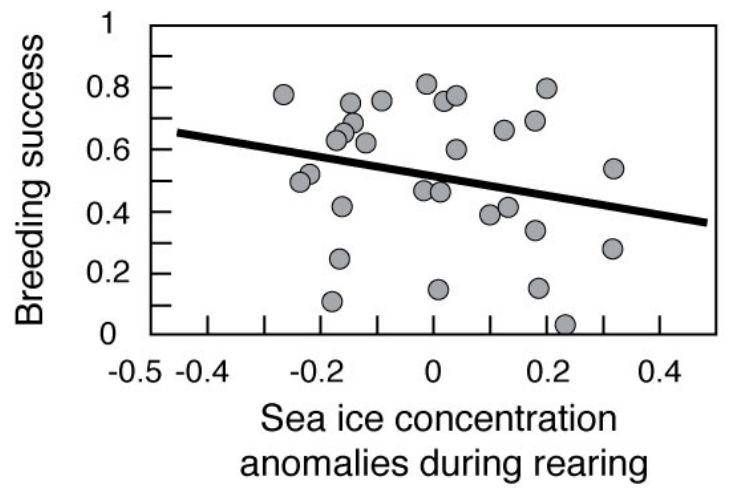

Figure 3: Breeding success as a function of proportional anomalies in SIC during the rearing season. The line is a logistic regression fit to the data points shown. 


\section{Females}

(a)

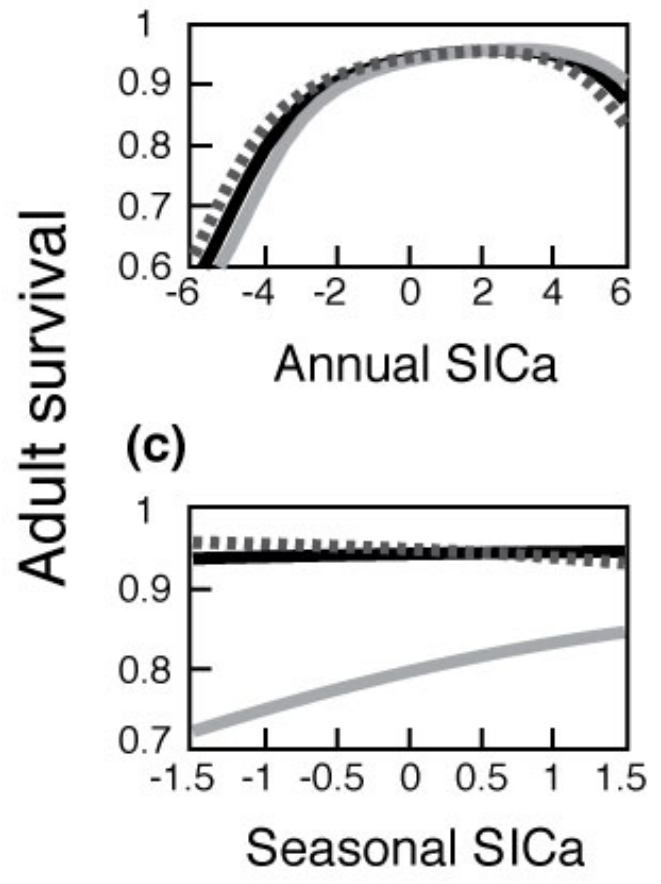

Males

(b)

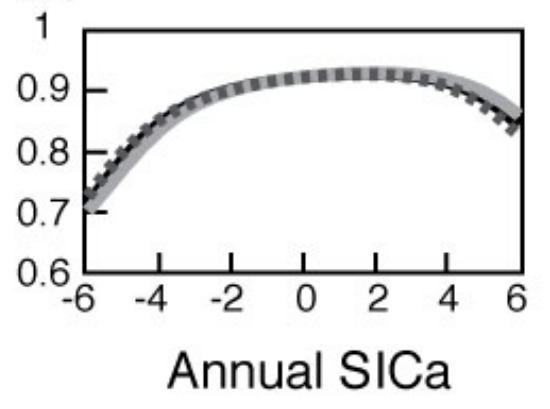

(d)

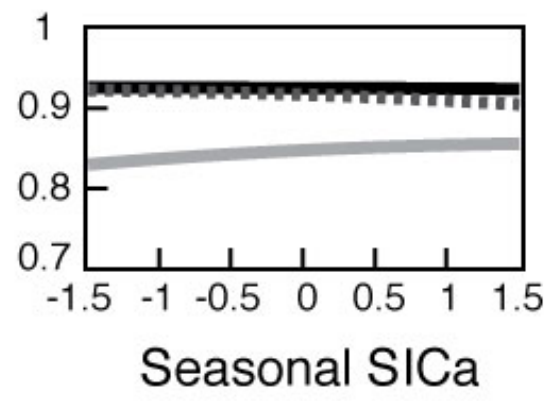

Figure 4: Annual adult survival as a function of annual $S I C_{a}$ and seasonal differences in $S I C_{a}$. (a c) and (b d) show the survival of females and males respectively. Upper panels (a b) show survival as a function of annual $S I C_{a}$ for negative $(=-1$, grey line), zero (thick black line), and positive $\left(=+1\right.$, dotted line) values of seasonal differences in $S I C_{a}$. Lower panels (c d) show survival as a function of seasonal differences in $S I C_{a}$ for negative (=-4, grey line), zero (thick black line), and positive $\left(=+4\right.$, dotted line) values of annual $S I C_{a}$. 


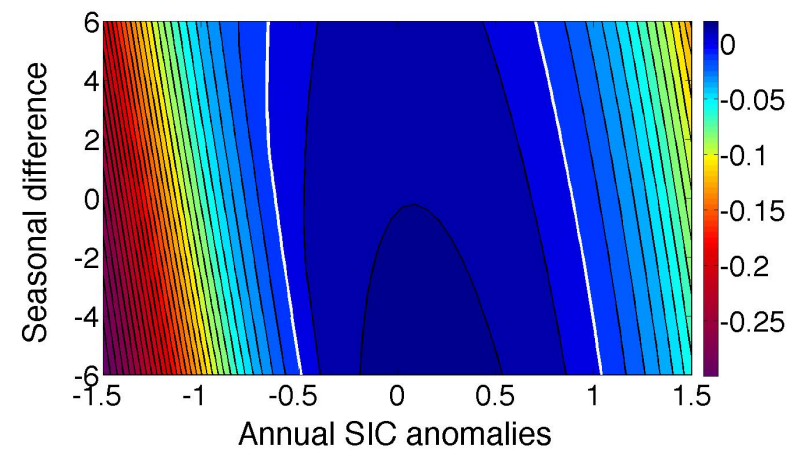

Figure 5: The deterministic population growth rate $(\log \lambda)$ as a function of annual $S I C_{a}$ and seasonal differences in $S I C_{a}$. The white contours indicate $\log \lambda=0$. The color bar shows the values of $\log \lambda$ 

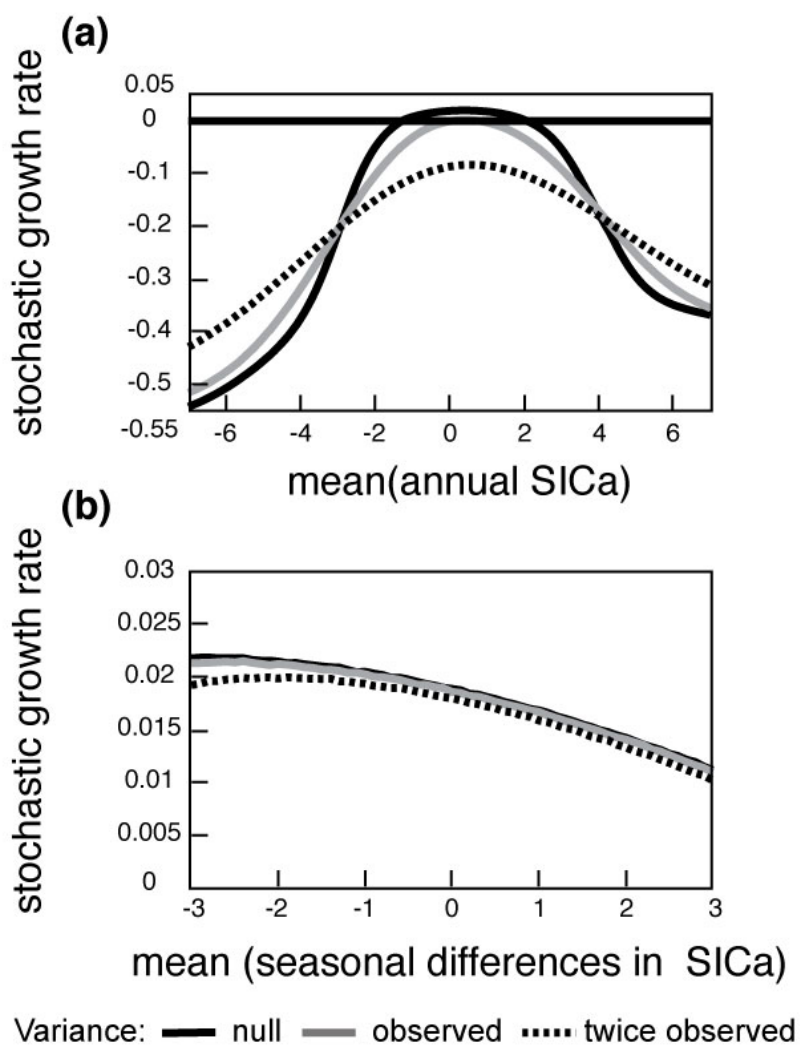

Figure 6: The stochastic population growth rate $\log \lambda_{s}$ as a function of the mean and variance of sea ice variables. (a) $\log \lambda_{s}$ as a function of the mean and variance of annual $S I C_{a}$ with seasonal differences in $S I C_{a}$ set to zero. The black line stands for a zero variance, the grey line for the observed variance, and the dotted line for a variance equal to twice the observed. (b) $\log \lambda_{s}$ as a function of the mean and variance of seasonal differences in $S I C_{a}$, with annual $S I C_{a}$ equal to zero. 


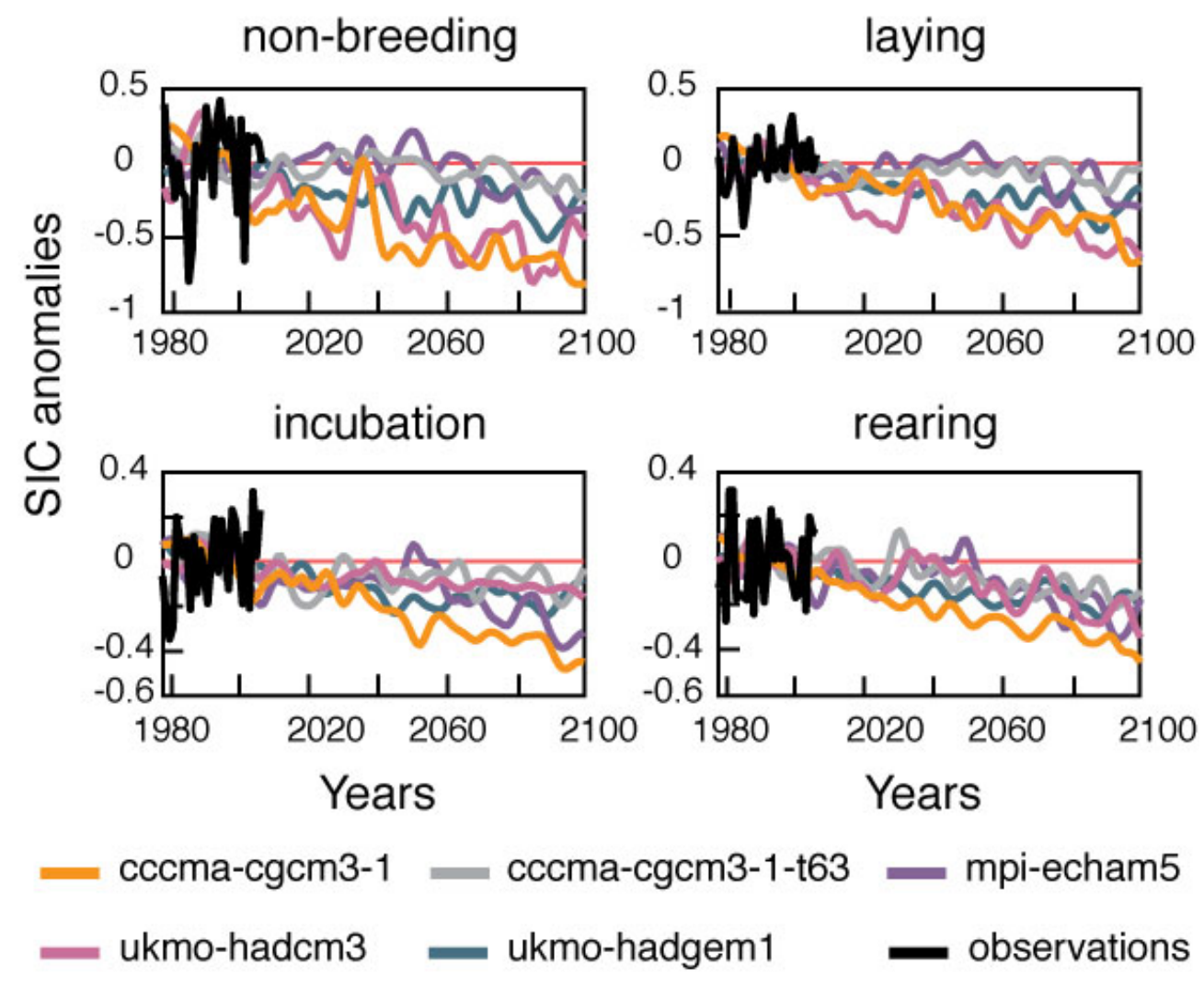

Figure 7: Smoothed mean of $S I C_{a}$ during the four seasons, from each of the five selected GCMs (color lines, see legend ). The black line shows the observed $S I C_{a}$ from 1979 to 2007 and the red line shows the zero value, i.e. represents averaged SIC from 1979 to 2007. 

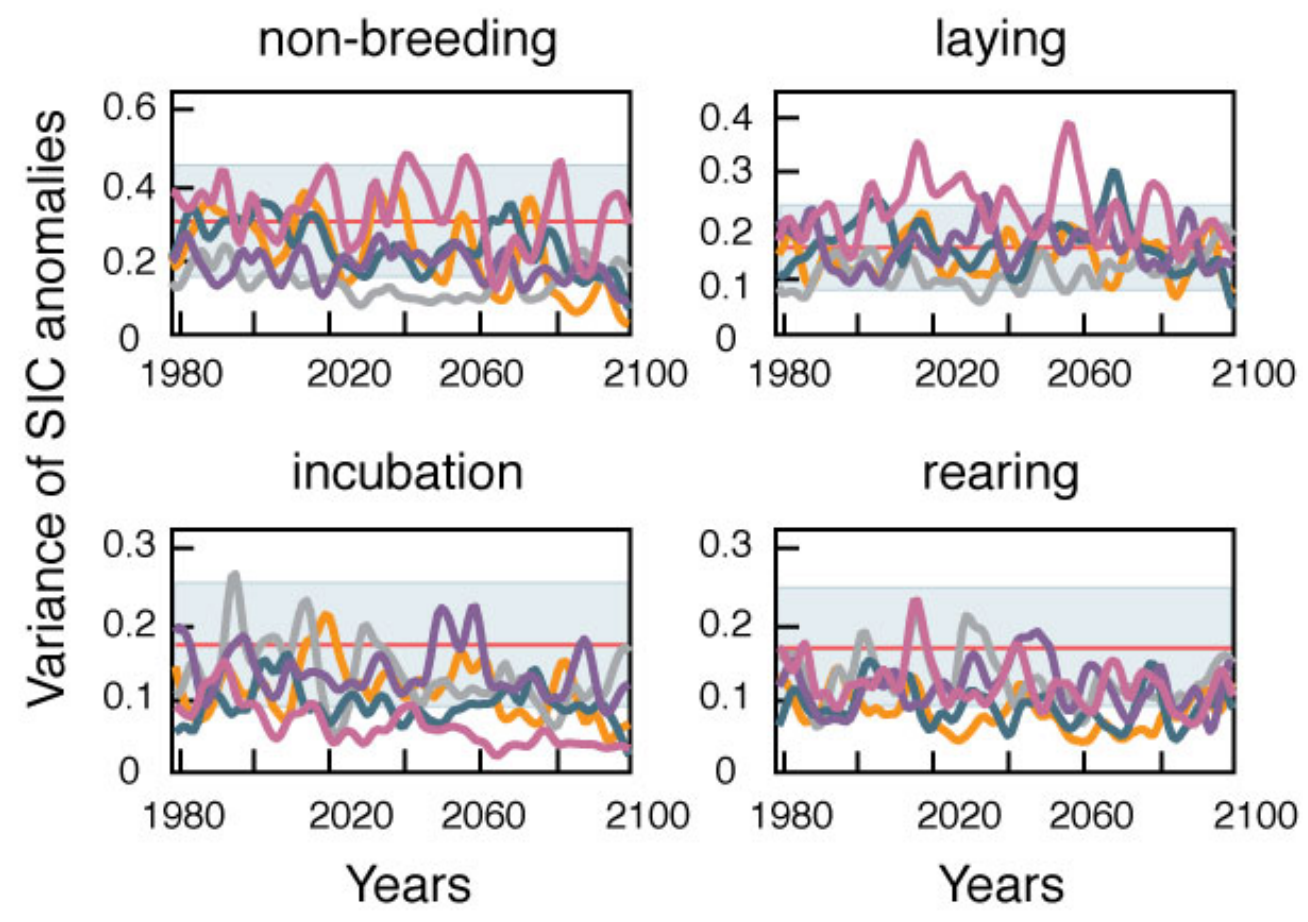

Figure 8: Smoothed standard deviation of $S I C_{a}$ during the four seasons, from each of the five selected GCMs (color lines, see legend on figure 7). The red line shows the observed standard deviation. The grey box represents 0.5 and 1.5 times the observed standard deviation; these values were used in selecting climate models (Appendix 4). 

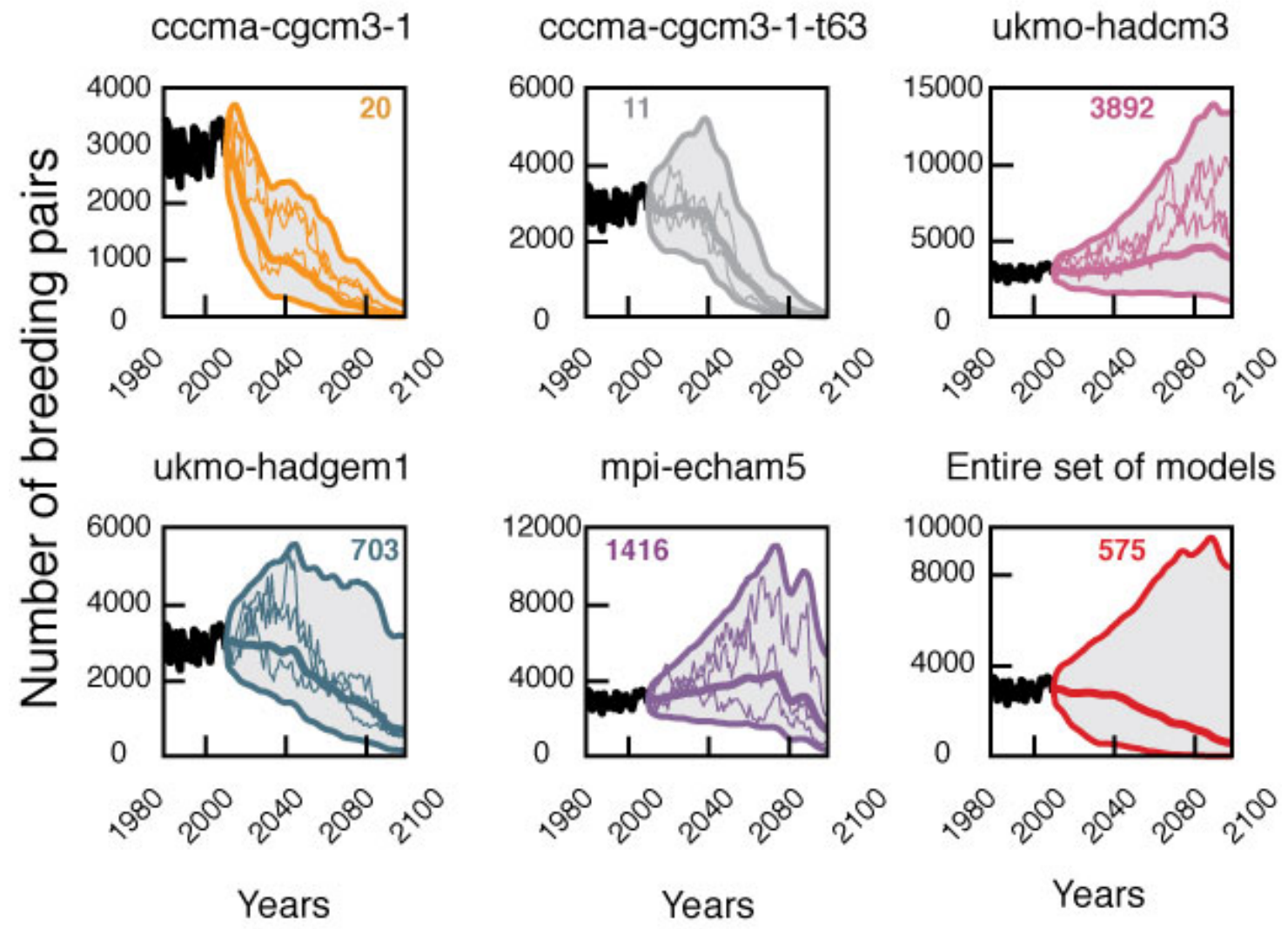

Years

Figure 9: Projections of the emperor penguin population based on $S I C_{a}$ forecasts from an ensemble of five GCMs. The black line gives the observed number of breeding pairs from 1979 to 2010. For each GCM, three random population trajectories are shown (thin colored lines), along with the median (thick colored line) and the 95\% envelope (grey area), from 40,000 stochastic simulations. The median and $95 \%$ envelope are also shown from the combined 200,000 simulations for the set of 5 GCMs. 


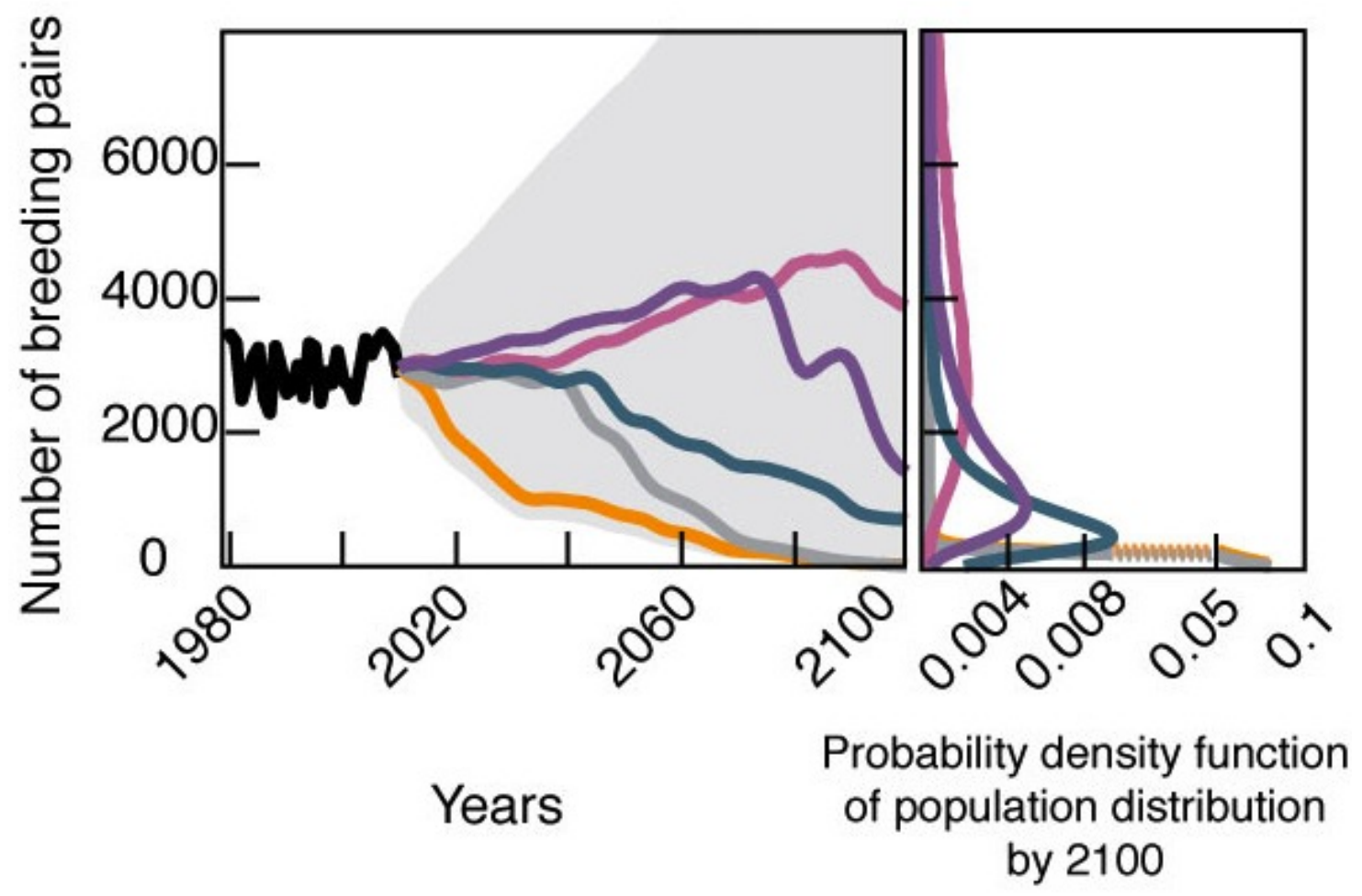

Figure 10: Summary of the projections of the emperor penguin population based on $S I C_{a}$ forecasts from an ensemble of five GCMs. The thick colored lines (see legend on figure 9) give the median and the grey area is the $95 \%$ envelope from the combined 200,000 simulations for the set of 5 GCMs. Also shown are the probability density functions for simulated population size in 2100 , for each GCM. 